\title{
Sliding joints in 3D beams: Conserving algorithms using the master-slave approach
}

\author{
José J. Muñoz • Gordan Jelenić
}

\begin{abstract}
This paper proposes two time-integration algorithms for motion of geometrically exact 3D beams under sliding contact conditions. The algorithms are derived using the socalled master-slave approach, in which constraint equations and the related time-integration of a system of differential and algebraic equations are eliminated by design. Specifically, we study conservation of energy and momenta when the sliding conditions on beams are imposed and discuss their algorithmic viability. Situations where the contact jumps to adjacent finite elements are analysed in detail and the results are tested on two representative numerical examples. It is concluded that an algorithmic preservation of kinematic constraint conditions is of utmost importance.
\end{abstract}

Keywords Master-slave method · Conserving time-integration · Sliding contact $\cdot$ Large rotations $\cdot 3 \mathrm{D}$ beams

\section{Motivation and introduction}

When dealing with frictionless sliding contact, it is a common practice to introduce either a potential function associated with the contact violation (penalty method, [2, 9]), or Lagrange multipliers which enforce the kinematic conditions of the contact $[4,9,17,26]$. The former brings forth the problem of choosing a problem-dependent penalty factor, which should be neither too large (ill conditioned system of equations) nor too low (excessive constraint violation). The latter approach introduces additional degrees of freedom (the Lagrange multipliers) and requires special techniques for the time-integration of the resulting differential-algebraic equations.

\footnotetext{
J. J. Muñoz

Department of Applied Mathematics III, Universitat Politècnica de Catalunya, Barcelona, Spain e-mail: j.munoz@upc.edu

G. Jelenić

University of Rijeka, Department of Civil Engineering, Rijeka, Republic of Croatia e-mail: gordan@gradri.hr
} 
We resort here to the so-called master-slave method, where the use of constraint equations is avoided by embedding the relation between the contact points into the discretised virtual work equation [10, 11, 19, 21] or into the energy increment [14]. In most of the previous works dealing with the master-slave approach, $[10,11,14,19,21]$, or the related discrete null-space method [5], the coordinates of the slave node are related to those of the reference master node and the relative released coordinates. This approach will be termed the pointto-point or node-to-node (NN) master-slave approach. Motivated by the desire to provide an exact sliding contact description of a node along a deformable beam or slideline, this relationship was modified in [21], where the slave degrees of freedom of one node were related to all the nodal displacements of the nodes of a master element. This approach will be called the node-to-element (NE) master-slave approach.

So far sliding joints have not been modelled in the literature using the master-slave approach and conserving time-integration algorithms. Other algorithms with conserving properties and sliding contact have been developed in $[2-4,16]$. References $[2,16]$ deal with contact problems in elastodynamics using a penalty method or augmented Lagrangian technique. References [3, 4] use Lagrange multipliers with 3D beams in combination with energy conservation. We also note that a similar technique to the NE master-slave approach has been recently used in [18] in the context of a 2D spring onto a rod, although no reference to conserving time-integration was done.

In the present work we aim at developing a substantially different technique from that presented in [3,4] by extending our earlier results [21] to an incrementally based conservative formulation. In this way, we use the minimal number of degrees of freedom and do not require any problem-dependent parameters. We discuss the obstacles we meet in pursuing this goal, study the compatibility of the conservation of angular momentum with the contact conditions, critically assess the priorities in meeting these requirements when it turns out to be difficult or impossible to satisfy both and, as a result, propose the following two algorithms: (i) a momentum conserving algorithm with relaxed sliding conditions, and (ii) a non-conserving algorithm which satisfies the contact conditions exactly.

\section{Beam theory and incremental weak form}

\subsection{Geometrically exact beam theory}

Let us parametrise each point $X$ of an homogeneous beam of density $\rho$ and length $L$ at a time $t$, by the two following mappings [24]: (i) the position vector of the centroid axis $\boldsymbol{r}(X, t):[0, L] \times \mathbb{R}_{+} \rightarrow \mathbb{R}^{3}$, and (ii) the rotation of the (undeformable) cross-section of the beam, represented by a rotation matrix $\Lambda(X, t):[0, L] \times \mathbb{R}_{+} \rightarrow S O(3)$. We define the reference configuration of the beam as a straight beam with its longer dimension aligned with the unit vector $\boldsymbol{e}_{1}$ of the inertial frame $\boldsymbol{e}_{i}$, and the two principal axis of the crosssection aligned with $\boldsymbol{e}_{2}$ and $\boldsymbol{e}_{3}$. By setting at each reference point $X$ an orthogonal triad $\boldsymbol{G}_{i}$ parallel to $\boldsymbol{e}_{i}$, the matrix $\boldsymbol{\Lambda}$ rotates the (fixed) triad $\boldsymbol{G}_{i}$ into the current (moving) frame $\mathbf{g}_{i}=\Lambda \boldsymbol{G}_{i}$.

It can be deduced that the material strain measures conjugate to the material stress and stress-couple resultants $\boldsymbol{N}$ and $\boldsymbol{M}$, are given by $\boldsymbol{\Gamma}=\boldsymbol{\Lambda}^{\mathrm{T}} \boldsymbol{r}^{\prime}-\boldsymbol{G}_{1}$ and $\boldsymbol{K}$, respectively [24], where here and henceforth the dash symbol $\left({ }^{\prime}\right)$ denotes differentiation with respect to the arclength parameter $X$, and vector $\boldsymbol{K}$ is obtained from $\boldsymbol{\Lambda}^{\prime}=\boldsymbol{\Lambda} \widehat{\boldsymbol{K}}$, where the hat $(\widehat{\bullet})$ onto a vector $\boldsymbol{a} \in \mathbb{R}^{3}$ denotes the skew-symmetric matrix such that $\widehat{\boldsymbol{a}} \boldsymbol{b}$ is the vector product $\boldsymbol{a} \times \boldsymbol{b}=-\widehat{\boldsymbol{b}} \boldsymbol{a}$. 
It follows from the previous description that the total elastic energy of the beam is given by

$$
V=\frac{1}{2} \int_{L}(\boldsymbol{N} \cdot \boldsymbol{\Gamma}+\boldsymbol{M} \cdot \boldsymbol{K}) d X
$$

In addition, we set $\mathbf{J}=\operatorname{diag}\left[\begin{array}{lll}I_{X} & I_{Y} & I_{X}+I_{Y}\end{array}\right]$ as the material tensor of second moments of area, and $\boldsymbol{W}$ as the material angular velocity such that $\dot{\Lambda}=\boldsymbol{\Lambda} \widehat{W}$, where the dot () stands for the time differentiation. With this notation, and the assumption of undeformability of the cross-section, the total kinetic energy of the beam is written as [25]

$$
T=\frac{1}{2} \int_{L} \rho(A \dot{\boldsymbol{r}} \cdot \dot{\boldsymbol{r}}+\boldsymbol{W} \cdot \mathbf{J W}) d X
$$

where $A$ is the cross-section area. Consequently, in the absence of material damping, the total energy $E$ of the beam is $E=V+T-W$, where $W$ is the work done by the external loads.

The beam differential equations of motion are derived in [24]. It can be verified that for conservative external loads, the change of the total energy $\Delta E$ is zero. In addition, the vectors of linear and angular momenta are both constant if no external loads exist [25]. We will introduce in the next section spatial and time discretisations that algorithmically conserve linear and angular momenta. This will make the basis for the application to problems with sliding contact.

\subsection{A momentum conserving invariant algorithm (MI)}

\subsubsection{Energy increment}

In order to simplify the subsequent derivations, we will assume that all the external forces are constant and all the external torques are zero. We will consider a deformed configuration of a beam at time $t_{n}$ determined by the position vector $\boldsymbol{r}_{n}(X)$ and the rotation matrix $\boldsymbol{\Lambda}_{n}(X)$ at each point $X$ of the centroid line, where henceforth $\{\bullet\}_{n}$ denotes quantities computed at time $t_{n}$, and $\{\bullet\}_{n+\frac{1}{2}}=\frac{1}{2}\left(\{\bullet\}_{n}+\{\bullet\}_{n+1}\right)$.

We define the incremental displacement $\boldsymbol{u}=\boldsymbol{r}_{n+1}-\boldsymbol{r}_{n}$, and the spatial and material incremental rotations $\boldsymbol{\omega}$ and $\boldsymbol{\Omega}$ between times $t_{n}$ and $t_{n+1}$, which are such that $\boldsymbol{\Lambda}_{n+1}=$ $\exp \widehat{\boldsymbol{\omega}} \boldsymbol{\Lambda}_{n}=\boldsymbol{\Lambda}_{n} \exp \widehat{\boldsymbol{\Omega}}$.

Also, we introduce the transformation matrix $\mathbf{H}=\mathbf{H}(\boldsymbol{\theta})=\mathbf{I}+\frac{1-\cos \theta}{\theta^{2}} \widehat{\boldsymbol{\theta}}+\frac{1}{\theta^{2}}\left(1-\frac{\sin \theta}{\theta}\right)$ $\widehat{\boldsymbol{\theta}}^{2}$ that satisfies the relation $\boldsymbol{\Lambda}_{n}^{\prime}=\widehat{\mathbf{H}(\boldsymbol{\omega}) \boldsymbol{\omega}^{\prime}} \boldsymbol{\Lambda}_{n}$, [7]. By setting $\omega=\|\boldsymbol{\omega}\|$, using the relations $[13,20] \boldsymbol{\Lambda}_{n+1}-\boldsymbol{\Lambda}_{n}=\frac{\tan (\omega / 2)}{\omega / 2} \widehat{\boldsymbol{\omega}} \boldsymbol{\Lambda}_{n+\frac{1}{2}}$ and $\boldsymbol{K}_{n+1}=\boldsymbol{K}_{n}+\boldsymbol{\Lambda}_{n}^{\mathrm{T}} \mathbf{H}(\boldsymbol{\omega})^{\mathrm{T}} \boldsymbol{\omega}^{\prime}$, and resorting to the definitions of the elastic and kinetic energy (1) and (2), we can write the increment of energy over a time step $\Delta t$ as $\Delta E=E_{n+1}-E_{n}=\Delta E=\Delta T+\Delta V-\Delta W$, where the increments can be expressed as [20]

$$
\begin{aligned}
& \Delta T=\frac{1}{\Delta t} \int_{L} \rho\left[A \boldsymbol{r}_{n+\frac{1}{2}} \cdot\left(\dot{\boldsymbol{u}}_{n+1}-\dot{\boldsymbol{u}}_{n}\right)+\boldsymbol{W}_{n+1} \cdot \mathbf{J}_{n+1} \boldsymbol{W}_{n+1}-\boldsymbol{W}_{n} \cdot \mathbf{J}_{n} \boldsymbol{W}_{n}\right] d X \\
& \Delta V=\int_{L}\left(\boldsymbol{u} \cdot \boldsymbol{\Lambda}_{n+\frac{1}{2}} \boldsymbol{N}_{n+\frac{1}{2}}+\frac{\tan (\omega / 2)}{\omega / 2} \widehat{\boldsymbol{r}}_{n+\frac{1}{2}}^{\prime} \boldsymbol{\omega} \cdot \boldsymbol{\Lambda}_{n+\frac{1}{2}} \boldsymbol{N}_{n+\frac{1}{2}}+\boldsymbol{\omega}^{\prime} \cdot \mathbf{H}(\boldsymbol{\omega}) \boldsymbol{\Lambda}_{n} \boldsymbol{M}_{n+\frac{1}{2}}\right) d X
\end{aligned}
$$




$$
\Delta W=\int_{L} \boldsymbol{u} \cdot \mathbf{n} d X
$$

and $\mathbf{n}$ is the external distributed force vector.

\subsubsection{Finite-element and time-discretisation}

Let us introduce a finite-element interpolation of the incremental quantities $\boldsymbol{u}$ and $\boldsymbol{\omega}$ as (from here on a summation over repeated superscript-subscript pair of indices will be understood)

$$
\boldsymbol{p}^{h}=\left\{\begin{array}{c}
\boldsymbol{u}^{h}(X) \\
\boldsymbol{\omega}^{h}(X)
\end{array}\right\}=I^{i}(X) \boldsymbol{p}_{i},
$$

where $\boldsymbol{p}_{i}^{\mathrm{T}}=\left\{\boldsymbol{u}_{i}^{\mathrm{T}} \boldsymbol{\omega}_{i}^{\mathrm{T}}\right\}$ are the nodal vectors of incremental displacements and rotations for each of the $N_{I}$ nodes of element $I$. Here, $I^{i}$ are the standard Lagrangian interpolation functions which satisfy the conditions $\sum_{i=1}^{N_{I}} I^{i}(X)=1$ and $I^{i}\left(X_{j}\right)=\delta_{j}^{i}$ (Kronecker delta). Using the following time-stepping

$$
\dot{\boldsymbol{r}}_{n+\frac{1}{2}}=\frac{\dot{\boldsymbol{r}}_{n+1}+\dot{\boldsymbol{r}}_{n}}{2}=\frac{\boldsymbol{u}}{\Delta t}, \quad \boldsymbol{W}_{n+\frac{1}{2}}=\frac{\boldsymbol{W}_{n+1}+\boldsymbol{W}_{n}}{2}=\frac{\boldsymbol{\Omega}}{\Delta t},
$$

and inserting the interpolation (4) in (3), the energy increment of a beam element over a time-step $\Delta t$ can be written as

$$
\Delta E \approx p_{I} \cdot g^{I}
$$

where $\boldsymbol{p}_{I}^{\mathrm{T}}=\left\{\boldsymbol{p}_{I, 1}^{\mathrm{T}} \ldots \boldsymbol{p}_{I, N_{I}}^{\mathrm{T}}\right\}$ is the transpose of the global vector of incremental kinematics with dimensions $6 \times N_{I}$, and $\boldsymbol{g}^{I^{\mathrm{T}}}=\left\{\boldsymbol{g}^{I, 1^{\mathrm{T}}} \ldots \boldsymbol{g}^{I, N_{I}^{\mathrm{T}}}\right\}$ is the transpose of the global residual vector $\boldsymbol{g}^{I}=\boldsymbol{g}_{d}^{I, i}+\boldsymbol{g}_{v}^{I, i}-\boldsymbol{g}_{\text {ext }}^{I, i}$, with the dynamic, elastic and external parts given by

$$
\begin{aligned}
& \boldsymbol{g}_{d}^{I, i}=\frac{1}{\Delta t} \int_{L} \rho I^{i}\left\{\begin{array}{c}
A\left(\dot{\boldsymbol{u}}_{n+1}-\dot{\boldsymbol{u}}_{n}\right) \\
\boldsymbol{\Lambda}_{n+1} \mathbf{J}_{n+1} \boldsymbol{W}_{n+1}-\boldsymbol{\Lambda}_{n} \mathbf{J}_{n} \boldsymbol{W}_{n}
\end{array}\right\} d X, \\
& \boldsymbol{g}_{v}^{I, i}=\int_{L}\left[\begin{array}{cc}
I^{i^{\prime} \mathbf{I}} & \mathbf{0} \\
-I^{i} \widehat{\boldsymbol{r}}_{n+\frac{1}{2}}^{\prime} & I^{i^{\prime} \mathbf{I}}
\end{array}\right]\left\{\begin{array}{c}
\boldsymbol{\Lambda}_{n+\frac{1}{2}} \boldsymbol{N}_{n+\frac{1}{2}} \\
\mathbf{T}(\boldsymbol{\omega})^{-1} \boldsymbol{\Lambda}_{n} \boldsymbol{M}_{n+\frac{1}{2}}
\end{array}\right\} d X, \\
& \boldsymbol{g}_{e}^{I, i}=\int_{L}\left\{\begin{array}{c}
I^{i} \overline{\mathbf{n}} \\
\mathbf{0}
\end{array}\right\} d X .
\end{aligned}
$$

The quantities in these expressions may be evaluated using interpolation (4), but this would spoil objectivity of the formulation with respect to the change of observer [12]. In order to retain this important physical property, the rotations need to be interpolated differently, using the material local rotations $\Theta^{h}=I^{i} \Theta_{i}$, which at time-step $t_{n}$ are defined by

$$
\Lambda_{n}^{h}=\Lambda_{\text {rig }, n} \exp \left(\widehat{\Theta}_{n}^{h}\right),
$$

with $\boldsymbol{\Lambda}_{r i g, n}$ the reference triad rigidly attached to the element. The fact that this interpolation is not the same as the interpolation of $\boldsymbol{\omega}$ in (4) (which plays the role of the rotational test 
function in $\Delta E$ ) and the factor $\frac{\tan (\omega / 2)}{\omega / 2}$ in (3b) are the reasons why the energy increment in (6) is not exactly equal to $\boldsymbol{p}_{I} \cdot \boldsymbol{g}^{I}$. Nevertheless it can be demonstrated that the present algorithm is exactly momentum conserving [20].

When considering a single element, the exact conservation of momenta and the approximate conservation of energy $\Delta E \approx 0$ are obtained for arbitrary incremental kinematics if the following system of non-linear equations is satisfied:

$$
\mathbf{g}^{I, i}=\mathbf{0}, \quad i=1, \ldots, N_{I} .
$$

For multi-element analysis, a similar system can be obtained by assembling all the elemental residual vectors $\boldsymbol{g}^{I^{\mathrm{T}}}=\left\{\boldsymbol{g}^{I, 1^{\mathrm{T}}} \ldots \boldsymbol{g}^{I, N_{I}^{\mathrm{T}}}\right\}$ in a standard finite-element manner.

\section{Master-slave relationship}

\subsection{Definition of a coupling element}

We will at first restrict our attention to the two-element assembly in Figure 1. We will name the elements A and B in this figure the slave and the master element, respectively. After the proposed spatial and time discretisation from the previous section has been applied, the deformed configurations of the beam elements at time $t_{n}$ are described via a set of nodal incremental displacement and rotation vectors $\boldsymbol{p}_{A, i}$ and $\boldsymbol{p}_{B, j}$. For the purposes of notational convenience, the sliding node will be taken to be the end node $N_{A}$. The point of its contact with element B at times $t_{n}$ and $t_{n+1}$ is determined by the arc-length coordinates $X_{n}$ and $X_{n+1}$, respectively.

Let us also define the vector of released displacements and rotations $\boldsymbol{p}_{R}^{\mathrm{T}}=\left\{\boldsymbol{u}_{R}^{\mathrm{T}} \boldsymbol{\omega}_{R}^{\mathrm{T}}\right\}$. The vectors $\boldsymbol{u}_{R}$ and $\boldsymbol{\omega}_{R}$ are obtainable from the following relations:

$$
\begin{aligned}
\boldsymbol{u}_{R}^{A, N_{A}} & =\left\{\begin{array}{lll}
X_{n+1}-X_{n} & 0 & 0
\end{array}\right\}^{\mathrm{T}} \\
\boldsymbol{\Lambda}_{n}^{A, N_{A}} & =\boldsymbol{\Lambda}_{X_{n}}^{B} \boldsymbol{\Lambda}_{R, n} \boldsymbol{\Lambda}_{r e l} \\
\boldsymbol{\Lambda}_{n+1}^{A, N_{A}} & =\boldsymbol{\Lambda}_{X_{n+1}}^{B} \boldsymbol{\Lambda}_{R, n+1} \boldsymbol{\Lambda}_{r e l} \\
\exp \left(\widehat{\boldsymbol{\omega}}_{R}\right) & =\boldsymbol{\Lambda}_{R, n+1} \boldsymbol{\Lambda}_{R, n}^{\mathrm{T}}
\end{aligned}
$$

where $\Lambda_{X_{n}}^{B}=\Lambda^{B}\left(X_{n}\right)$ and $\Lambda_{X_{n+1}}^{B}=\Lambda^{B}\left(X_{n+1}\right)$ are the rotation matrices of the master element $B$ at the contact points $X_{n}$ and $X_{n+1}$ (at times $t_{n}$ and $t_{n+1}$, respectively), and matrix $\boldsymbol{\Lambda}_{r e l}$ is a constant relative rotation matrix between the beams at the contact point in the initial configuration i.e, at $t=0, \Lambda_{X_{0}}^{A}=\Lambda_{X_{0}}^{B} \Lambda_{r e l}$. The matrix $\Lambda_{R}$ is the matrix of released rotation, that is the exponential of the (material) rotation between the orientation of the cross section

Fig. 1 Two-element model of a sliding node. Nodes on master and slave elements are represented by white and black circles, respectively
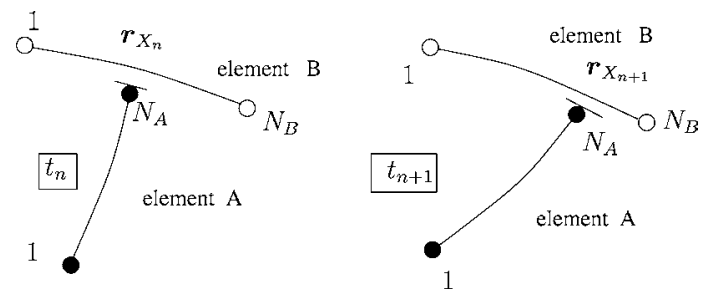
of beam B at the contact point rotated by $\boldsymbol{\Lambda}_{\text {rel }}$, and the orientation of the cross section at node $N_{A}$. The aim of the master-slave approach is to replace the incremental displacements and rotations of the slave node $\boldsymbol{p}_{A, N_{A}}$, by a set of master and released degrees of freedom, $\boldsymbol{p}_{A, R m}$, which can be regarded as the degrees of freedom of a coupling element. The latter vector contains the incremental displacements and rotations $\boldsymbol{p}_{R}$, all the nodal slave incremental displacements and rotations $\boldsymbol{p}_{A}^{\mathrm{T}}=\left\{\boldsymbol{p}_{A, 1}^{\mathrm{T}} \ldots \boldsymbol{p}_{A, N_{A}}^{\mathrm{T}}\right\}$ and the nodal incremental displacements and rotations of the master element $B$ :

$$
\boldsymbol{p}_{A, R m}^{\mathrm{T}}=\left\{\boldsymbol{p}_{R}^{\mathrm{T}} \boldsymbol{p}_{A, 1}^{\mathrm{T}} \cdots \boldsymbol{p}_{A, N_{A}}^{\mathrm{T}} \boldsymbol{p}_{B, 1}^{\mathrm{T}} \ldots \boldsymbol{p}_{B, N_{B}}^{\mathrm{T}}\right\}
$$

In the following section we will derive the explicit expression of a transformation matrix $\mathbf{N}$ such that

$$
\boldsymbol{p}_{A}=\mathbf{N} \boldsymbol{p}_{A, R m} .
$$

\subsection{Displacements}

We will denote the position vectors of the contact points by $\boldsymbol{r}_{X_{n}}=\boldsymbol{r}\left(X_{n}\right)$ and $\boldsymbol{r}_{X_{n+1}}=\boldsymbol{r}\left(X_{n+1}\right)$ at times $t_{n}$ and $t_{n+1}$ respectively, both of them located on element $B$. Situations where the contact point $\boldsymbol{r}_{n+1}$ is located within an adjacent element will be studied in Section 5 .

Using the Lagrangian interpolation functions $I^{j}$ on the master element $B$, the sliding kinematic conditions can be written as follows:

$$
\begin{array}{ll}
\underline{t_{n}}: & \boldsymbol{r}_{A, N_{A}, n}=\boldsymbol{r}_{X_{n}}=I_{X_{n}}^{j} \boldsymbol{r}_{j, n} \\
\underline{t_{n+1}}: & \boldsymbol{r}_{A, N_{A}, n+1}=\boldsymbol{r}_{X_{n+1}}=I_{X_{n+1}}^{j} \boldsymbol{r}_{j, n+1}
\end{array}
$$

with $\quad \boldsymbol{r}_{A, N_{A}, n}=\boldsymbol{r}\left(X_{A, N_{A}}, t_{n}\right), \quad \boldsymbol{r}_{A, N_{A}, n+1}=\boldsymbol{r}\left(X_{A, N_{A}}, t_{n+1}\right), \quad I_{X_{n}}^{j}=I^{j}\left(X_{n}\right)$, and $I_{X_{n+1}}^{j}=$ $I^{j}\left(X_{n+1}\right)$.

It is helpful to have at hand the diagram in Figure 2. It gives an insight into the position of the contact point in the two sliding situations mentioned above by representing deformed configurations where no horizontal displacements of the master nodes exist. In this case, the $x$ axis of the figure is representative of the arc-length coordinate $X$. The deformed configuration at a mid-time $t_{n+\frac{1}{2}}$, which for the master nodes is given by $\boldsymbol{r}_{n+\frac{1}{2}}=I^{j} \boldsymbol{r}_{j, n+\frac{1}{2}}$, is also depicted in the figure.

Note first that two different paths from $\boldsymbol{r}_{X_{n}}$ to $\boldsymbol{r}_{X_{n+1}}$ can be observed in Figure 2, one through point $\mathrm{P}$ and another through point $\mathrm{Q}$. Both paths can be combined via a weighting parameter $\gamma$, leading to the following general expression:

$$
\begin{aligned}
\boldsymbol{u}_{A, N_{A}} & =(1-\gamma)\left(\boldsymbol{u}_{t_{n}}+\boldsymbol{u}_{X_{n+1}}\right)+\gamma\left(\boldsymbol{u}_{X_{n}}+\boldsymbol{u}_{t_{n+1}}\right) \\
& =\left((1-\gamma) \boldsymbol{u}_{t_{n}}+\gamma \boldsymbol{u}_{t_{n+1}}\right)+\left(\gamma \boldsymbol{u}_{X_{n}}+(1-\gamma) \boldsymbol{u}_{X_{n+1}}\right),
\end{aligned}
$$

where the meaning of $\boldsymbol{u}_{t_{n}}, \boldsymbol{u}_{t_{n+1}}, \boldsymbol{u}_{X_{n}}$ and $\boldsymbol{u}_{X_{n+1}}$ is illustrated in Figure 2, and defined as follows:

$$
\begin{aligned}
\boldsymbol{u}_{t_{n}}=\Delta I_{X}^{j} \boldsymbol{r}_{j, n}, & \boldsymbol{u}_{t_{n+1}}=\Delta I_{X}^{j} \boldsymbol{r}_{j, n+1}, \\
\boldsymbol{u}_{X_{n}}=I_{X_{n}}^{j} \boldsymbol{u}_{j}, & \boldsymbol{u}_{X_{n+1}}=I_{X_{n+1}}^{j} \boldsymbol{u}_{j},
\end{aligned}
$$




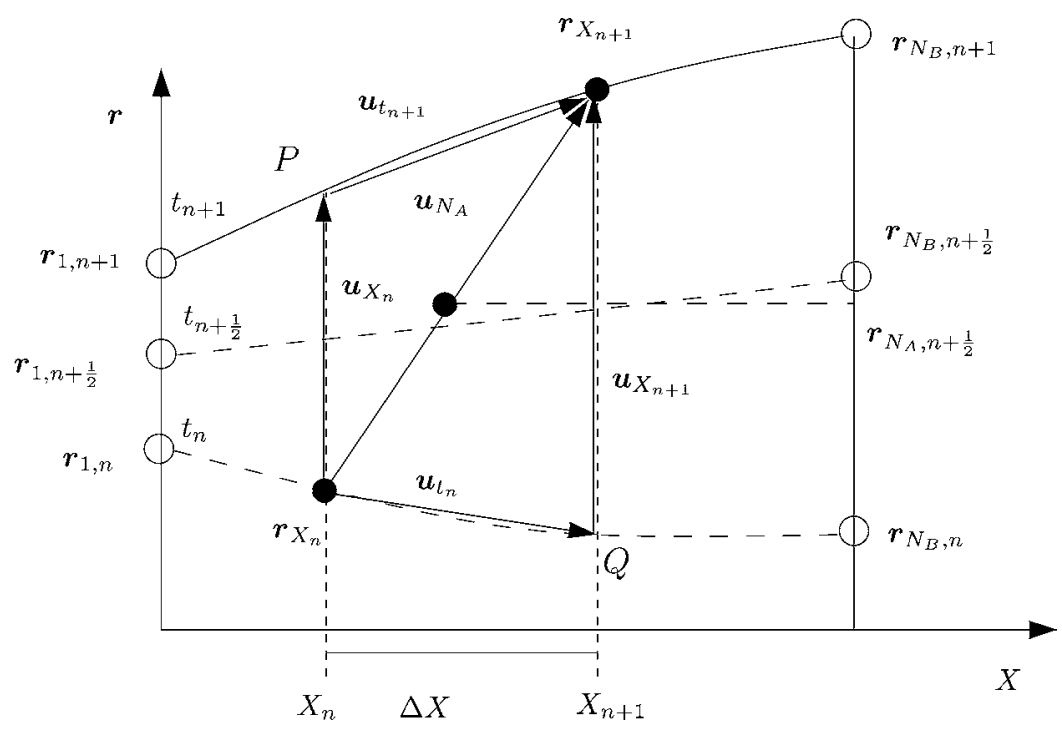

Fig. 2 Displacement increments over one time-step within one element

with $\Delta I_{X}^{j}=I_{X_{n+1}}^{j}-I_{X_{n}}^{j}$. For $\gamma=0$ and $\gamma=1$ the paths via points Q and P are recovered, respectively. By setting

$$
\begin{aligned}
I_{\gamma}^{j}(X) & =\gamma I_{X_{n}}^{j}+(1-\gamma) I_{X_{n+1}}^{j}, \\
\boldsymbol{u}_{n, \gamma} & =\gamma \boldsymbol{u}_{t_{n}}+(1-\gamma) \boldsymbol{u}_{t_{n+1}},
\end{aligned}
$$

we can rewrite (15) as

$$
\boldsymbol{u}_{A, N_{A}}=\boldsymbol{u}_{n, 1-\gamma}+I_{\gamma}^{j}(X) \boldsymbol{u}_{j}
$$

Noting that $\Delta X=\boldsymbol{u}_{R} \cdot \boldsymbol{G}_{R}$, with $\boldsymbol{G}_{R}^{\mathrm{T}}=\boldsymbol{G}_{1}^{\mathrm{T}}=\left\{\begin{array}{lll}1 & 0 & 0\end{array}\right\}$ as the material unit vector in the direction of the released displacement, the vector $\boldsymbol{u}_{n, 1-\gamma}$ can be written as $\boldsymbol{u}_{n, 1-\gamma}=\frac{\boldsymbol{u}_{n, 1-\gamma}}{\Delta X} \Delta X=$ $\frac{1}{\Delta X}\left(\boldsymbol{u}_{n, 1-\gamma} \otimes \boldsymbol{G}_{1}\right) \boldsymbol{u}_{R}$, which inserted into (18) leads to

$$
\boldsymbol{u}_{A, N_{A}}=\frac{1}{\Delta X}\left(\boldsymbol{u}_{n, 1-\gamma} \otimes \boldsymbol{G}_{1}\right) \boldsymbol{u}_{R}+I_{\gamma}^{j}(X) \boldsymbol{u}_{j} .
$$

We emphasise that, in writing these expressions, no approximation other than the standard FE interpolation has been introduced.

\subsection{Rotations}

Keeping in mind the two-element model in Figure 1, we will deduce first a relationship between the incremental nodal rotations of the master element $B$ and those of the slave element $A$ at node $N_{A}$. Let us write the contact conditions at times $t_{n}$ and $t_{n+1}$ as follows:

$$
\begin{aligned}
\boldsymbol{\Lambda}_{A, N_{A}, n} & =\boldsymbol{\Lambda}_{X_{n}} \boldsymbol{\Lambda}_{R, n} \boldsymbol{\Lambda}_{r e l} \\
\boldsymbol{\Lambda}_{A, N_{A}, n+1} & =\boldsymbol{\Lambda}_{X_{n+1}} \boldsymbol{\Lambda}_{R, n+1} \boldsymbol{\Lambda}_{r e l}
\end{aligned}
$$


The incremental slave rotation $\boldsymbol{\omega}_{A, N_{A}}$, the incremental released rotation $\boldsymbol{\omega}_{R}$ and the incremental master rotations $\boldsymbol{\omega}_{X}$ are defined by $\exp \left(\widehat{\boldsymbol{\omega}}^{A, N_{A}}\right)=\boldsymbol{\Lambda}_{A, N_{A}, n+1} \boldsymbol{\Lambda}_{A, N_{A}, n}^{\mathrm{T}} ; \exp \left(\widehat{\boldsymbol{\omega}}_{R}\right)=$ $\boldsymbol{\Lambda}_{R, n+1} \boldsymbol{\Lambda}_{R, n}^{\mathrm{T}}$, and $\exp \left(\widehat{\boldsymbol{\omega}}_{X}\right)=\boldsymbol{\Lambda}_{X_{n+1}}^{B} \boldsymbol{\Lambda}_{X_{n}}^{B}$. Thus, $\boldsymbol{\omega}_{X}$ is the incremental rotation from $t_{n}$ to $t_{n+1}$ of the triad attached to the cross-section of the contact point. It is useful to work with the tangent-scaled counterparts of these incremental rotations, defined by $\underline{\omega}=\frac{\tan (\omega / 2)}{\omega / 2} \boldsymbol{\omega}$. It can be verified that the parametrisation of rotations with tangent-scaled vectors leads to the Cayley transform [1]:

$$
\exp (\widehat{\boldsymbol{\omega}})=\operatorname{cay}(\underline{\boldsymbol{\omega}})=\mathbf{I}+\frac{1}{1+\frac{1}{4} \underline{\omega}^{2}}\left(\underline{\widehat{\boldsymbol{\omega}}}+\frac{1}{2} \underline{\widehat{\omega}}\right) .
$$

It follows then that the tangent-scaled rotation vectors $\underline{\omega}_{A, N_{A}}, \underline{\omega}_{R}$ and $\underline{\omega}_{X}$ are such that

$$
\begin{aligned}
\boldsymbol{\Lambda}_{A, N_{A}, n+1} & =\operatorname{cay}\left(\underline{\boldsymbol{\omega}}_{A, N_{A}}\right) \boldsymbol{\Lambda}_{A, N_{A}, n}, \\
\boldsymbol{\Lambda}_{R, n+1} & =\operatorname{cay}\left(\underline{\boldsymbol{\omega}}_{R}\right) \boldsymbol{\Lambda}_{R, n}, \\
\boldsymbol{\Lambda}_{X_{n+1}} & =\operatorname{cay}\left(\underline{\boldsymbol{\omega}}_{X}\right) \boldsymbol{\Lambda}_{X_{n}} .
\end{aligned}
$$

Inserting relations (21) into (20b), and making use of the contact condition (20a), yields

$$
\begin{aligned}
\operatorname{cay}\left(\underline{\boldsymbol{\omega}}_{A, N_{A}}\right) \boldsymbol{\Lambda}_{A, N_{A}, n} & =\operatorname{cay}\left(\underline{\boldsymbol{\omega}}_{X}\right) \boldsymbol{\Lambda}_{X_{n}} \operatorname{cay}\left(\underline{\boldsymbol{\omega}}_{R}\right) \boldsymbol{\Lambda}_{R, n} \boldsymbol{\Lambda}_{r e l} \\
& =\operatorname{cay}\left(\underline{\boldsymbol{\omega}}_{X}\right) \operatorname{cay}\left(\boldsymbol{\Lambda}_{X_{n}} \underline{\boldsymbol{\omega}}_{R}\right) \boldsymbol{\Lambda}_{A, N_{A}, n},
\end{aligned}
$$

from where cay $\left(\underline{\omega}_{A, N_{A}}\right)=\operatorname{cay}\left(\underline{\omega}_{X}\right) \operatorname{cay}\left(\Lambda_{X_{n}} \underline{\omega}_{R}\right)$.

We will mimic the displacement field shown in Figure 2 by splitting the incremental rotation $\underline{\omega}_{X}$ (which is due to the increments $\Delta X$ and $\Delta t$ ) into two parts,

$$
\operatorname{cay}\left(\underline{\omega}_{X}\right)=\operatorname{cay}\left(\underline{\omega}_{X_{n+1}}\right) \operatorname{cay}\left(\underline{\omega}_{t_{n}}\right),
$$

and therefore we can write

$$
\begin{aligned}
\boldsymbol{\Lambda}_{X_{n+1}, t_{n}} & =\operatorname{cay}\left(\underline{\boldsymbol{\omega}}_{t_{n}}\right) \boldsymbol{\Lambda}_{X_{n}, t_{n}} \\
\boldsymbol{\Lambda}_{X_{n+1}, t_{n+1}} & =\operatorname{cay}\left(\underline{\boldsymbol{\omega}}_{X_{n+1}}\right) \boldsymbol{\Lambda}_{X_{n+1}, t_{n}} .
\end{aligned}
$$

Figure 3 explains the meaning of the incremental rotations $\underline{\omega}_{t_{n}}$ and $\underline{\omega}_{X_{n+1}}$. The former is the rotation between points $X_{n}$ and $X_{n+1}$ with the time fixed at $t_{n}$, whereas the latter is the incremental rotation of the cross-section at point $X_{n+1}$ of the master element $B$. Note that the released rotation $\Lambda_{R}$, also to be considered, is not represented in the figure, and the relation in (23) corresponds to the path $\boldsymbol{\Lambda}_{X_{n}}-\boldsymbol{\Lambda}_{Q}-\boldsymbol{\Lambda}_{X_{n+1}}$. The relationship deduced here is general for any kind of rotation. Depending on the number of free released components of the rotational vector $\omega_{R}$, different joints may be obtained. For instance, for the cylindrical, revolute, Cardan and spherical joints, vector $\boldsymbol{\omega}_{R}$ has one, one, two or three non-zero components, respectively.

Inserting Equation (23) into (22) we get

$$
\operatorname{cay}\left(\underline{\boldsymbol{\omega}}_{A, N_{A}}\right) \boldsymbol{\Lambda}_{A, N_{A}, n}=\operatorname{cay}\left(\underline{\boldsymbol{\omega}}_{X_{n+1}}\right) \operatorname{cay}\left(\underline{\boldsymbol{\omega}}_{t_{n}}\right) \operatorname{cay}\left(\boldsymbol{\Lambda}_{X_{n}} \underline{\boldsymbol{\omega}}_{R}\right) \boldsymbol{\Lambda}_{A, N_{A}, n},
$$

which implies that $\underline{\boldsymbol{\omega}}_{A, N_{A}}=\underline{\boldsymbol{\omega}}_{X_{n+1}} \circ \underline{\boldsymbol{\omega}}_{t_{n}} \circ\left(\boldsymbol{\Lambda}_{X_{n}} \underline{\boldsymbol{\omega}}_{R}\right)$ (composition of three rotations). A formula for the compound rotation of three successive rotations can be derived using the 


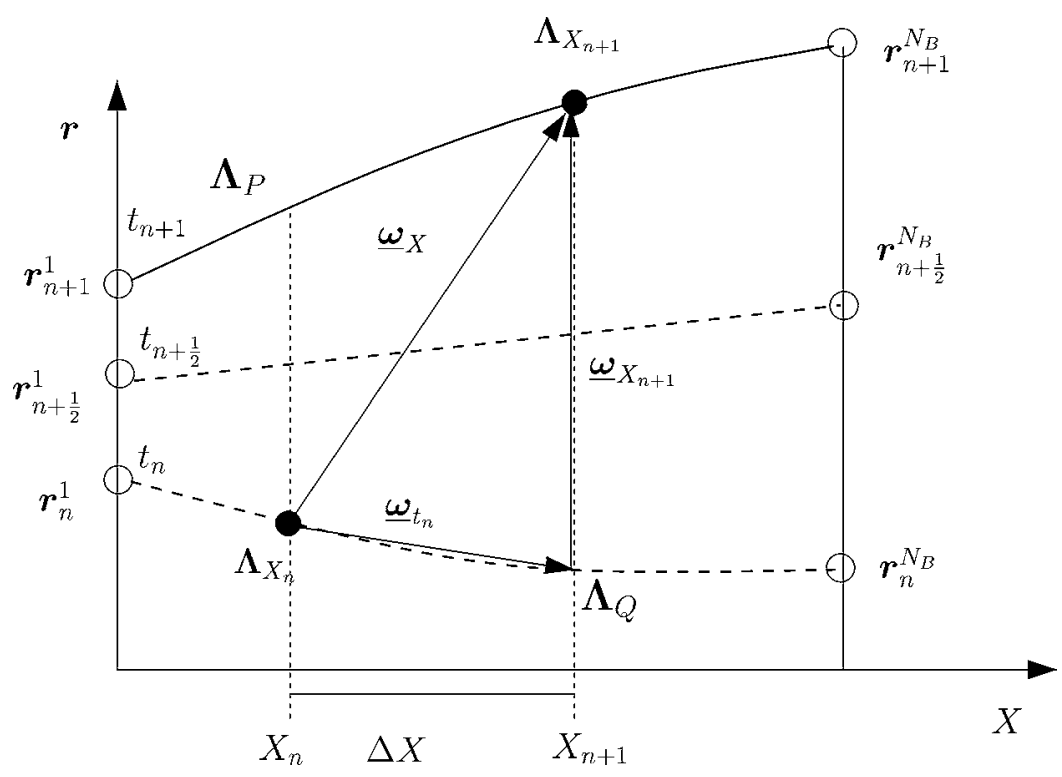

Fig. 3 Rotational increments over a single time-step

expression of the compound rotation of two rotations [1], which in our case may be written in the general form

$$
\underline{\boldsymbol{\omega}}_{A, N_{A}}=\mathbf{A} \underline{\boldsymbol{\omega}}_{X_{n+1}}+\mathbf{B} \underline{\boldsymbol{\omega}}_{t_{n}}+\mathbf{C} \Lambda_{X_{n}} \underline{\boldsymbol{\omega}}_{R}
$$

where

$$
\begin{aligned}
\mathbf{A}= & \mathbf{I}, \mathbf{B}=z \mathbf{S}\left(\underline{\boldsymbol{\omega}}_{X_{n+1}}\right)^{\mathrm{T}}, \quad \mathbf{C}=z \mathbf{S}\left(\underline{\boldsymbol{\omega}}_{X_{n+1}}\right)^{\mathrm{T}}\left(\mathbf{I}+\frac{1}{2} \widehat{\widehat{\omega}}_{t_{n}}\right), \\
z^{-1}= & 1-\frac{1}{4}\left(\underline{\boldsymbol{\omega}}_{t_{n}} \cdot \boldsymbol{\Lambda}_{X_{n}} \underline{\boldsymbol{\omega}}_{R}+\underline{\boldsymbol{\omega}}_{X_{n+1}} \cdot \underline{\boldsymbol{\omega}}_{t_{n}}+\underline{\boldsymbol{\omega}}_{X_{n+1}} \cdot \boldsymbol{\Lambda}_{X_{n}} \underline{\boldsymbol{\omega}}_{R}\right) \\
& -\frac{1}{8} \underline{\boldsymbol{\omega}}_{X_{n+1}} \cdot \underline{\widehat{\omega}}_{t_{n}} \Lambda_{X_{n}} \underline{\boldsymbol{\omega}}_{R},
\end{aligned}
$$

and the matrix $\mathbf{S}(\underline{\omega})$ is given by $\mathbf{S}(\underline{\omega})=\boldsymbol{I}-\frac{1}{2} \underline{\widehat{\omega}}+\frac{1}{4} \underline{\boldsymbol{\omega}} \otimes \underline{\boldsymbol{\omega}}$. It is shown in Appendix A that the identity $\mathbf{A}=\mathbf{I}$ is required for the conservation of angular momentum.

Using the interpolation of incremental tangent-scaled rotations $\underline{\omega}_{X_{n+1}}=I_{X_{n+1}}^{j} \underline{\omega}_{j}$, we can rewrite (25a) as

$$
\underline{\boldsymbol{\omega}}_{A, N_{A}}=I_{X_{n+1}}^{j} \underline{\boldsymbol{\omega}}_{j}+\frac{1}{\Delta X} \mathbf{B}\left(\underline{\boldsymbol{\omega}}_{t_{n}} \otimes \boldsymbol{G}_{1}\right) \boldsymbol{u}_{R}+\mathbf{C} \boldsymbol{\Lambda}_{X_{n}} \underline{\boldsymbol{\omega}}_{R}
$$

By resorting to the approximation $\frac{\tan (\omega / 2)}{\omega / 2} \approx 1$ for the nodal incremental slave and master rotations, Equation (26) can be written as:

$$
\boldsymbol{\omega}_{A, N_{A}} \approx I_{X_{n+1}}^{j} \boldsymbol{\omega}_{j}+\frac{1}{\Delta X} \mathbf{B}\left(\underline{\boldsymbol{\omega}}_{t_{n}} \otimes \boldsymbol{G}_{1}\right) \boldsymbol{u}_{R}+\mathbf{C} \boldsymbol{\Lambda}_{X_{n}} \underline{\boldsymbol{\omega}}_{R}
$$


The effects of the mentioned approximation in the resulting algorithm will be discussed in the next section. Note that the interpolation indicated in (27) will be used for the construction of the equilibrium equations, but that nodal rotations $\boldsymbol{\omega}_{j}$ and the rotation $\underline{\boldsymbol{\omega}}_{t_{n}}$ are in fact computed using the interpolation of material local rotations $\Theta_{j}$ as expressed in (8).

\section{Master-slave algorithms}

\subsection{Master-slave transformation matrix}

By gathering together relationships (19) and (27), we can provide the explicit form of matrix $\mathbf{N}$ in (13) as

$$
\mathbf{N}=\left[\begin{array}{cccccccc}
\overline{\mathbf{0}} & \overline{\mathbf{I}} & \ldots & \overline{\mathbf{0}} & \overline{\mathbf{0}} & \overline{\mathbf{0}} & \ldots & \overline{\mathbf{0}} \\
\vdots & \vdots & \ddots & \vdots & \vdots & \vdots & \ddots & \vdots \\
\overline{\mathbf{0}} & \overline{\mathbf{0}} & \ldots & \overline{\mathbf{I}} & \overline{\mathbf{0}} & \overline{\mathbf{0}} & \ldots & \overline{\mathbf{0}} \\
\mathbf{R} & \overline{\mathbf{0}} & \ldots & \overline{\mathbf{0}} & \overline{\mathbf{0}} & \overline{\mathbf{I}}_{X}^{1} & \ldots & \overline{\mathbf{I}}_{X}^{N_{B}}
\end{array}\right]
$$

where $\overline{\mathbf{I}}_{X}^{j}$ and $\mathbf{R}$ are given by

$$
\mathbf{R}=\left[\begin{array}{cc}
\frac{1}{\Delta X} \boldsymbol{u}_{n, 1-\gamma} \otimes \boldsymbol{G}_{1} & \mathbf{0} \\
\frac{1}{\Delta X} \mathbf{B}\left(\underline{\omega}_{t_{n}} \otimes \boldsymbol{G}_{1}\right) & \mathbf{C} \boldsymbol{\Lambda}_{X_{n}}
\end{array}\right], \quad \overline{\mathbf{I}}_{X}^{j}=\left[\begin{array}{cc}
I_{\gamma}^{j}(X) \mathbf{I} & \mathbf{0} \\
\mathbf{0} & I_{X_{n+1}}^{j} \mathbf{I}
\end{array}\right] .
$$

Here and henceforth, the matrices $\overline{\mathbf{I}}$ and $\overline{\mathbf{0}}$ are the identity and zero $6 \times 6$ matrices, respectively. Note that the vector $\boldsymbol{p}_{A, R m}$ in (13) now consists of the incremental released displacements and the incremental released tangent-scaled rotations.

\subsection{Joints with dependent degrees of freedom}

For the screw, rack-and-pinion and the cam joint, there exist two mutually dependent released degrees of freedom $[20,22]$. Within the current approach, this internal relationship can be taken into account by modifying the transformation matrix $\mathbf{N}$. Although we will only show its resulting expression for the screw joint, the details of the necessary modifications for other kinds of joints can be found in [20]. A similar manipulation was performed in [22] for the NN approach.

Given a screw joint with pitch $c$, the relation between the incremental released displacements $\Delta \boldsymbol{r}_{R}$ and the incremental released rotation $\omega_{R}\left(\right.$ or $\left.\underline{\omega}_{R}\right)$ can be written as

$$
\Delta \boldsymbol{r}_{R}=c \boldsymbol{\omega}_{R}=c \frac{\arctan (\underline{\omega} / 2)}{\underline{\omega} / 2} \underline{\omega}_{R}
$$

where the last identity has been introduced due to the fact that matrix $\mathbf{N}$ in (28) relates the slave incremental rotations to the tangent-scaled incremental released rotations $\underline{\omega}_{R}$. Inserting this relation into matrix $\mathbf{N}$ is tantamount to replacing matrix $\mathbf{R}$ in (28b) by,

$$
\mathbf{R}=\left[\begin{array}{cc}
\mathbf{0} & \frac{2 c \arctan (\underline{\omega} / 2)}{\underline{\omega} \Delta X} \boldsymbol{u}_{n, 1-\gamma} \otimes \boldsymbol{G}_{1} \\
\mathbf{0} & \frac{2 c \arctan (\underline{\omega} / 2)}{\underline{\omega} \Delta X} \mathbf{B}\left(\underline{\omega}_{t_{n}} \otimes \boldsymbol{G}_{1}\right)+\mathbf{C} \boldsymbol{\Lambda}_{X_{n}}
\end{array}\right] .
$$




\subsection{Equilibrium equations}

Considering the two-beam model depicted in Figure 1, the master-slave relationship $\boldsymbol{p}_{A}=$ $\mathbf{N} \boldsymbol{p}_{A, R m}$ can be inserted in the discretised expression of the energy increment $\Delta E$ in (6), which leads to

$$
\Delta E \approx \boldsymbol{p}_{A, R m} \cdot \mathbf{N}^{\mathrm{T}} \boldsymbol{g}^{A}+\boldsymbol{p}_{B} \cdot \boldsymbol{g}^{B} .
$$

The vector $\boldsymbol{g}_{R m}^{A}=\mathbf{N}^{\mathrm{T}} \boldsymbol{g}^{A}$ can be regarded as an extended residual vector of the coupling element, which is conjugate to the incremental displacements and rotations $\boldsymbol{p}_{A, R m}$. In our twoelement model, after assembling the vectors of the coupling element and the residual vector of element $B$, the following equilibrium equations are obtained by setting the right-hand side of the above equation to zero for arbitrary incremental kinematics in $\boldsymbol{p}_{A, R m}$ :

$$
\begin{array}{rl}
\mathbf{R}^{\mathrm{T}} \boldsymbol{g}^{A, N_{A}}=\mathbf{0} & \\
\mathbf{g}^{A, i}=\mathbf{0} & i=1, \ldots, N_{A}-1 \\
\boldsymbol{g}^{B, j}+\overline{\mathbf{I}}_{X \gamma}^{j} \boldsymbol{g}^{A, N_{A}}=\mathbf{0} \quad j=1, \ldots, N_{B} .
\end{array}
$$

Although the modelling of friction is out of the scope of this paper, let us observe that the extension of the method to account for this phenomenon would require computation of the relative displacements (or velocities) at the contact point and the contact forces. The former can be obtained directly from the released degrees of freedom, whereas the latter may be computed evaluating the load vector $\boldsymbol{g}^{A, N_{A}}$ in (30). We note that $\mathbf{R}^{\mathrm{T}} \boldsymbol{g}^{A, N_{A}}$ equals 0 (the projection of the load vector on the released degrees of freedom), but that $\boldsymbol{g}^{A, N_{A}}$ will be different from zero on the direction where contact exists.

\subsection{Properties of the algorithms}

While deriving Equation (6) and the master-slave relation for rotations in (27), we have used the approximation $1 \approx \frac{\tan (\omega / 2)}{\omega / 2}$. Unless $\omega \rightarrow 0$, this approximation will spoil the conservation of energy and, in fact, the more approximate the master-slave relationship, the larger will be the error in the energy conservation. The dual interpolation of rotations (4) and (8) additionally affects the energy conservation.

Regarding the conservation of momenta, it is shown in Appendix A that the increment of angular momentum over a time-step can be written as (see Equation (43b) with $\mathbf{A}=\mathbf{I}$ ):

$$
\Delta \boldsymbol{\Pi}=\frac{\Delta t}{2}\left(\gamma \widehat{\boldsymbol{u}}_{t_{n+1}}-(1-\gamma) \widehat{\boldsymbol{u}}_{t_{n}}\right) \boldsymbol{g}_{f}^{A, N_{A}},
$$

where $\boldsymbol{g}_{f}^{A, N_{A}}$ is the residual force vector at node $N_{A}$, and $\boldsymbol{u}_{t_{n}}$ and $\boldsymbol{u}_{t_{n+1}}$ are defined in (16) and in Figure 2. Therefore, the conservation of angular momentum requires the condition $\gamma \widehat{\boldsymbol{u}}_{t_{n+1}}-(1-\gamma) \widehat{\boldsymbol{u}}_{t_{n}}=\mathbf{0}$ to be satisfied. This can be also written as

$$
\gamma\left(\boldsymbol{u}_{t_{n+1}}+\boldsymbol{u}_{t_{n}}\right)=\boldsymbol{u}_{t_{n}}
$$

If the sliding contact conditions are satisfied, the error in identity (32) is in general reduced by setting $\gamma=\frac{1}{2}$. This fact leads us to construct the following two algorithms: 
- SMI algorithm. Conservation of momenta is maintained by satisfying identity (32). For the same reason, the contact kinematics (14) is violated. As a result, an additional error in the conservation of energy occurs. Both of these effects are minimised for $\gamma=\frac{1}{2}$. The position vector of the slave node is then computed from Equation (43a) as

$$
\boldsymbol{r}_{A, N_{A}, n+1}=2 I_{X \frac{1}{2}}^{j} \boldsymbol{r}_{j, n+\frac{1}{2}}^{B}-\boldsymbol{r}_{A, N_{A}, n} .
$$

Note that the interpolation matrices $\overline{\mathbf{I}}_{X}$ defined in (28b) remain unchanged. The different slave node kinematics affects the update process, but not the form of the equilibrium equations.

- SI algorithm. This algorithm is in some sense complementary to SMI: the contact kinematics (14) is upheld, but this prevents condition (32) for the conservation of momenta to be satisfied. No additional error is introduced in the conservation of energy.

The numerical examples given in Section 6 will show that the satisfaction of the sliding condition (which does not increase the energy increment) contributes importantly to the stable response of the algorithm. In addition, it will be shown that even though the SI algorithm is not exactly conserving, the incremental variations in energy and angular momentum remain comparatively limited.

\section{Contact transition}

Figure 4 illustrates the situation where the two contact points $X_{n+1}$ and $X_{n}$ lie on two different elements, and Figure 5 is the corresponding version of Figure 2 when contact transition occurs. Prior to writing a master-slave relationship, it is important to note that if the incremental kinematics of both master elements $B$ and $C$ would be inserted in the resulting coupling element, the linearisation of the equilibrium equations would couple all three elements $(A, B$ and $C$ ). Defining such a coupling element or, by induction, a coupling element with a slideline composed of an even larger number of master beam elements, is possible, but this would largely increase the computational cost. For this reason, our coupling element will always consist of one slave and one master beam element, of which the latter may be changeable. In the forthcoming derivations, therefore, we will relate the incremental kinematics of the slave element $A$ to the current master element $C$ at time $t_{n+1}$ (but not to element $B$ contacted at time $t_{n}$ ).

The previous argument implies the use of $\gamma=0$ in Equation (19) (path $\boldsymbol{r}_{X_{n}}-Q-\boldsymbol{r}_{X_{n+1}}$ in Figure 5), which leads to the following expression of the increment of the slave displacements:

$$
\boldsymbol{u}_{A, N_{A}}=\frac{1}{\Delta X}\left(\boldsymbol{u}_{t_{n}} \otimes \boldsymbol{G}_{r}\right) \boldsymbol{u}_{R}+I_{X_{n+1}}^{j} \boldsymbol{u}_{j}
$$
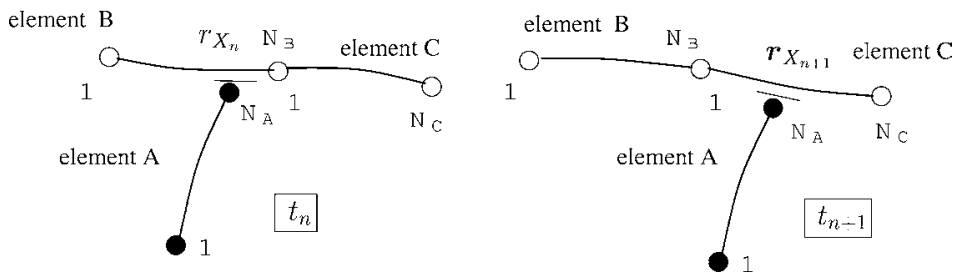

Fig. 4 Simplified model for problems with contact transition 


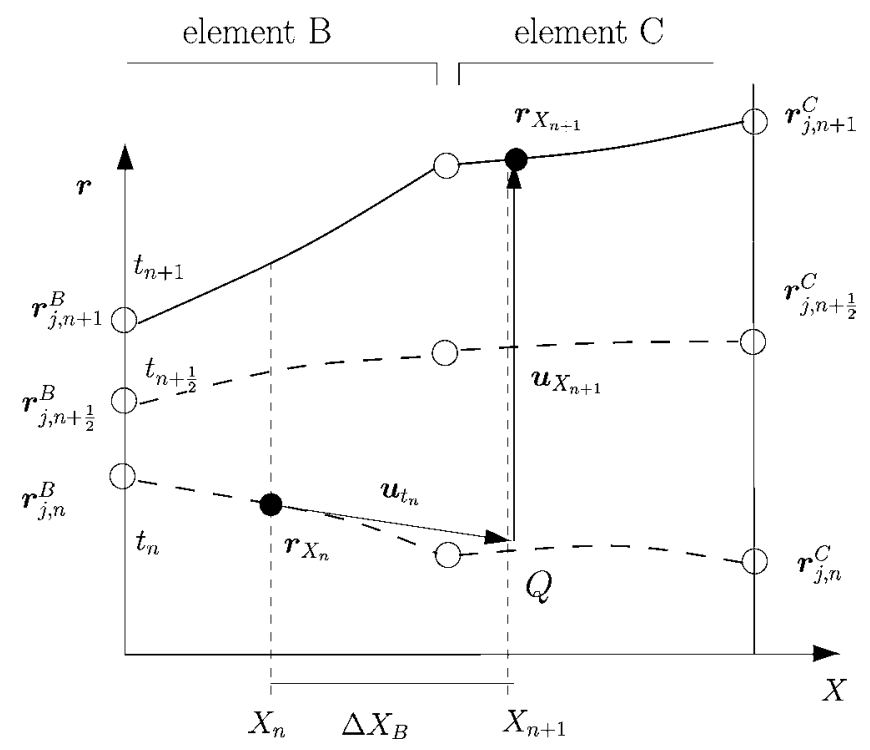

Fig. 5 Displacement increments over one time-step in the presence of contact transition

Note that now, since $\boldsymbol{r}_{t_{n}}\left(X_{n+1}\right)$ and $\boldsymbol{r}_{t_{n}}\left(X_{n}\right)$ belong to different elements, $\boldsymbol{u}_{t_{n}}$ is not given by $(16)_{1}$, but by (see Figure 5 )

$$
\boldsymbol{u}_{t_{n}}=I_{X_{n+1}}^{j} \boldsymbol{r}_{C, j, n}-I_{X_{n}}^{j} \boldsymbol{r}_{B, j, n} .
$$

Using similar manipulations to those in Appendix A, it can be shown that the increment of the angular momentum in the present case can be expressed as:

$$
\Delta \boldsymbol{\Pi}=\Delta t\left(\widehat{\boldsymbol{r}}_{A, N_{A}, n+\frac{1}{2}}-I_{X_{n+1}}^{j} \widehat{\boldsymbol{r}}_{C, j, n+\frac{1}{2}}\right) \boldsymbol{g}_{f}^{A, N_{A}} .
$$

As in the previous section, the equation $\widehat{\boldsymbol{r}}_{A, N_{A}, n+\frac{1}{2}}-I_{X_{n+1}}^{j} \widehat{\boldsymbol{r}}_{C, j, n+\frac{1}{2}}=\mathbf{0}$ is in general in contradiction with the kinematic conditions of a sliding joint, which are now written as

$$
\begin{array}{ll}
\underline{t_{n}}: & \boldsymbol{r}_{A, N_{A}, n}=\boldsymbol{r}_{X_{n}}=I_{X_{n}}^{j} \boldsymbol{r}_{B, j, n} \\
\underline{t_{n+1}}: & \boldsymbol{r}_{A, N_{A}, n+1}=\boldsymbol{r}_{X_{n+1}}=I_{X_{n+1}}^{j} \boldsymbol{r}_{C, j, n+1} .
\end{array}
$$

We can either satisfy the kinematic condition in (37) or conserve the angular momentum. Consistent with the choices given in the previous section, the SMI and SI algorithms will be completed using the following definitions:

- SMI algorithm. The angular momentum is satisfied, which requires the following kinematic condition:

$$
\boldsymbol{r}_{A, N_{A}, n+1}=2 I_{X_{n+1}}^{j} \boldsymbol{r}_{j, n+\frac{1}{2}}^{C}-\boldsymbol{r}_{A, N_{A}, n} .
$$

We point out that this is a coarser approximation of the sliding condition than relation (33) for the SMI algorithm described in the previous section, and therefore the conservation of 
Table 1 Summary of the conserving and kinematic properties of the SMI and SI algorithms

\begin{tabular}{llcr}
\hline & $\Delta \boldsymbol{\Pi}$ & Sliding condition & $\gamma$ \\
\hline SMI & $\mathbf{0}$ & $\boldsymbol{r}_{A, N_{A}, n+1}=$ & \\
& & $2 I_{X \frac{1}{2}}^{j} \boldsymbol{r}_{j, n+\frac{1}{2}}^{B}-\boldsymbol{r}_{A, N_{A}, n}$ & $\frac{1}{2}$ \\
$\mathrm{SI}$ & $\frac{\Delta t}{2}\left(\widehat{\boldsymbol{u}}_{t_{n+1}}-\widehat{\boldsymbol{u}}_{t_{n}}\right) \boldsymbol{g}_{f}^{A, N_{A}}$ & $\sqrt{ }$ & $\frac{1}{2}$ \\
$\mathrm{SMI}(\mathrm{T})$ & $\mathbf{0}$ & $\boldsymbol{r}_{A, N_{A}, n+1}=$ & 0 \\
& & $2 I_{X_{n+1}}^{j} \boldsymbol{r}_{j, n+\frac{1}{2}}^{C}-\boldsymbol{r}_{A, N_{A}, n}$ & \\
$\mathrm{SI}(\mathrm{T})$ & $-\frac{\Delta t}{2} \widehat{\boldsymbol{u}}_{t_{n}} \boldsymbol{g}_{f}^{A, N_{A}}$ & $\sqrt{ }$ & 0 \\
\hline
\end{tabular}

The properties of the algorithms for situations where contact transition occurs have been indicated by $(\mathrm{T})$

energy is yet additionally worsened. Numerical results will confirm this fact, and although this algorithm loses certain robustness during the contact transition, it has been introduced here for comparison reasons.

- SI algorithm. The kinematic conditions are satisfied (note that with the contact transition $\gamma$ has to be set to zero). The conserving properties are the same as for the SI algorithm without transition of the contact point between elements.

The increment of angular momentum in algorithm SI is obtained by inserting the kinematic condition (37) into expression (36), which gives rise to

$$
\Delta \boldsymbol{\Pi}=\frac{\Delta t}{2}\left(I_{X_{n}}^{j} \widehat{\boldsymbol{r}}_{B, j, n}-I_{X_{n+1}}^{k} \widehat{\boldsymbol{r}}_{C, k, n}\right)=-\frac{\Delta t}{2} \widehat{\boldsymbol{u}}_{t_{n}} \boldsymbol{g}_{f}^{A, N_{A}},
$$

where $\boldsymbol{u}_{t_{n}}$ is given in (35). Table 1 summarises the properties of the algorithms described so far. We note that the master-slave relationship for rotations in (27) does not need to be modified due to the contact transition.

\section{Numerical examples}

\subsection{Free sliding mass}

This example involves two flexible beams connected through a spherical sliding joint and serves to present the conservation properties of the proposed algorithms. The initial configuration and the co-ordinates of the two beam nodes are given in Figure 6. The geometrical and material properties are identical for both beams, except their lengths. A mass of $1 \mathrm{~kg}$ is attached to beam $B M$ at point $M$ and subjected to an initial velocity $v_{0}^{\mathrm{T}}=\left\{\begin{array}{lll}0 & -10-10\end{array}\right\}$. Since there exist no applied loads, the problem is genuinely energy and momentum conserving.

In all the following simulations, the beams $A B$ and $B M$ are discretised using four and one quadratic elements respectively. The simulations are run until the sliding node on beam $B M$ reaches point $A$. We tested the node-to-element (NE) approach to model the sliding joint using the trapezoidal rule and the SMI and SI algorithms. The application of the trapezoidal rule to sliding contact within the master-slave approach can be found in [21]. A 


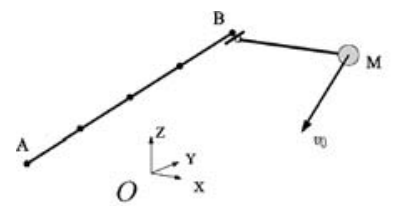

$$
\begin{aligned}
& E I_{y y}=E I_{z z}=20.0 \\
& \rho I_{y y}=\rho I_{z z}=0.016 \\
& A E=100.0 ; \rho A=0.08 ; \nu=0.3
\end{aligned}
$$

$$
\overline{O A}^{\mathrm{T}}=\left\{\begin{array}{llll}
0.0 & 0.0 & 0.0
\end{array}\right\} ; \overline{O B}^{\mathrm{T}}=\left\{\begin{array}{llll}
0.0 & 3.0 & 1.0
\end{array}\right\} ; \overline{O M}^{\mathrm{T}}=\left\{\begin{array}{lll}
1.0 & 3.0 & 1.0
\end{array}\right\}
$$

Fig. 6 Free sliding mass example

Fig. 7 Motion simulation for the free sliding mass problem using algorithm SI
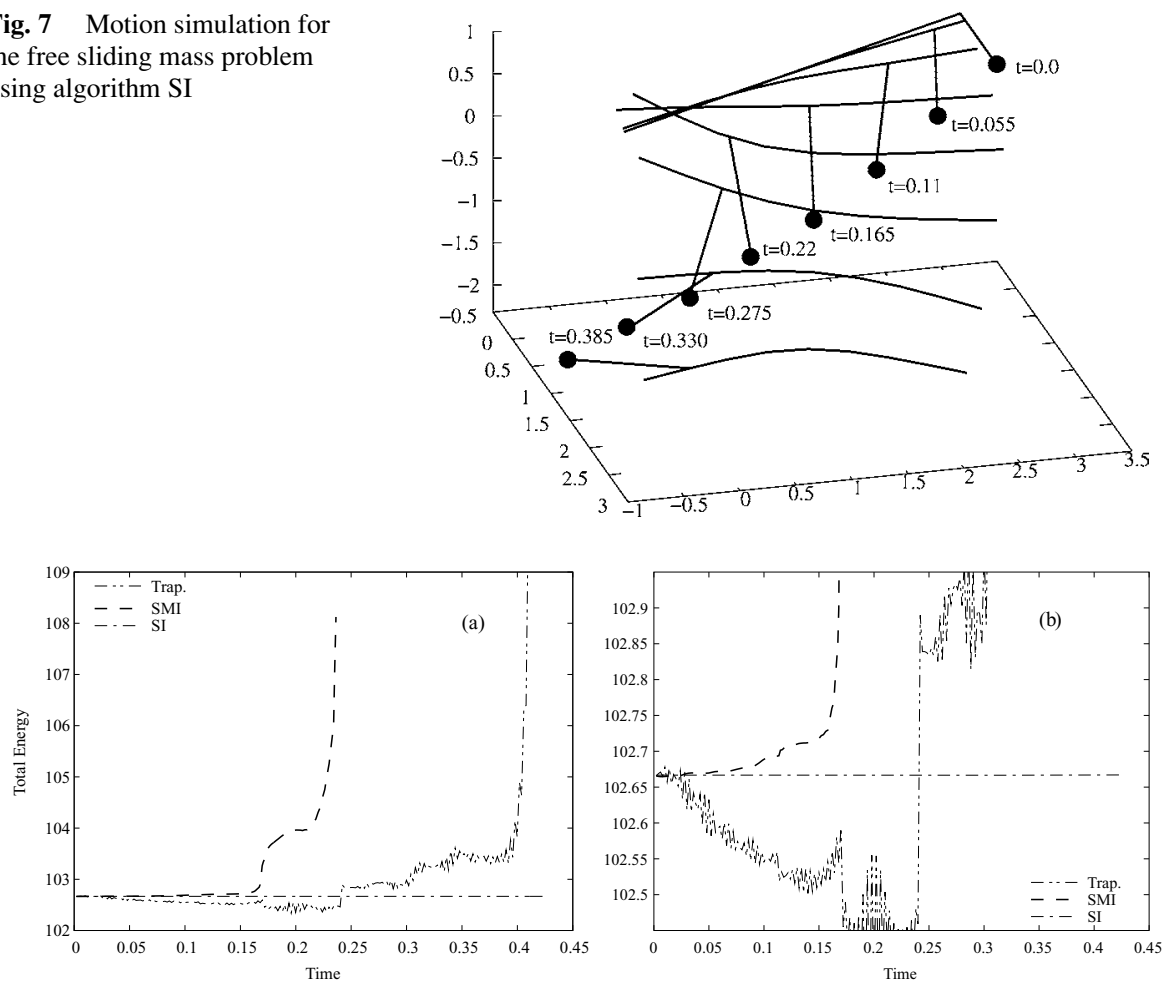

Fig. 8 Evolution of the total energy for the free sliding mass problem

series of deformed configurations at different times using the SI algorithm are depicted in Figure 7.

We applied the algorithms mentioned above with a constant time-step $\Delta t=0.002$. The algorithm SI ran the analysis successfully. The SMI algorithm failed to converge at time $t=0.23674$, after five successive time-step halvings. The same algorithm encountered also difficulties to converge just before the first two contact point transitions (times $t=0.1134$ and $t=0.1634$ ). At these times the time-step convergence was achieved by halving the time-step size, and then progressively increasing it until the initial time-step size was restored. This is a consequence of the approximated sliding kinematics which SMI uses in order to conserve the angular momentum. During the contact transition, this effect is additionally present.

The evolution of the total energy in Figure 8 confirms this reasoning, where the energy increments of the SMI algorithm become severe after the second contact transition. A similar 

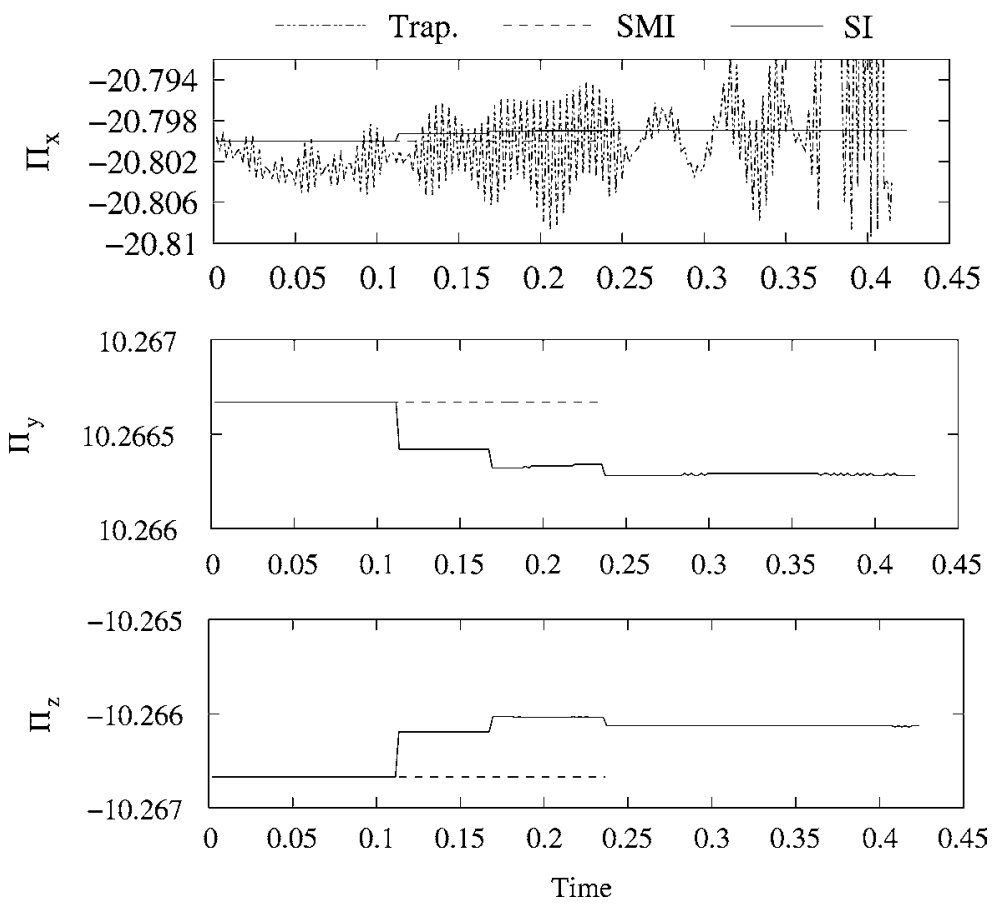

Fig. 9 Three components of the angular momentum for the trapezoidal rule, algorithm SMI and algorithm SI

behaviour is observed in the trapezoidal rule, although to a lesser extent (we recall that this algorithm satisfies the contact condition). Towards the end of the analysis, though, this algorithm develops an energy blow-up and eventually fails to converge before the slave node reaches the end A. Remarkably, the energy of algorithm SI has very small oscillations. We recall that these are due to the interpolation of local rotations and the approximations $1 \approx \frac{\tan (\omega / 2)}{\omega / 2}$ used in (6) and (27) while constructing the equilibrium equations.

Figure 9 shows the evolution of the angular momentum. The instabilities observed in the total energy evolution in algorithm SMI are not present in the angular momentum, which is kept constant. The trapezoidal rule has pronounced oscillations, as observed in the results for $\Pi_{x}$. The oscillations for the $\Pi_{y}$ and $\Pi_{z}$ components are of the same magnitude, but have not been plotted in the figure for clarity. Although SI does not conserve the angular momentum, its components remain apparently bounded. Larger variations in these components normally show up during the transition of the contact point between elements.

\subsection{Driven screw joint, Bauchau and Bottasso [4]}

In this example a vertical driver is attached to a fixed point $A$ through a revolute joint with its axis of rotation in the direction of $Z$ (see Figure 10). The other end $B$ is connected to a horizontal beam first through an universal (Cardan) joint where the only constrained rotation is the one along the axis of the horizontal beam, and afterwards through a screw joint with the released rotation along the same axis. The pitch of the screw is $c=\frac{2.4}{\pi / 3}=2.2918 \mathrm{~m} / \mathrm{rad}$ which corresponds to a twist of $60^{\circ}$ from point $R$ to point $T$. The beam is also physically 


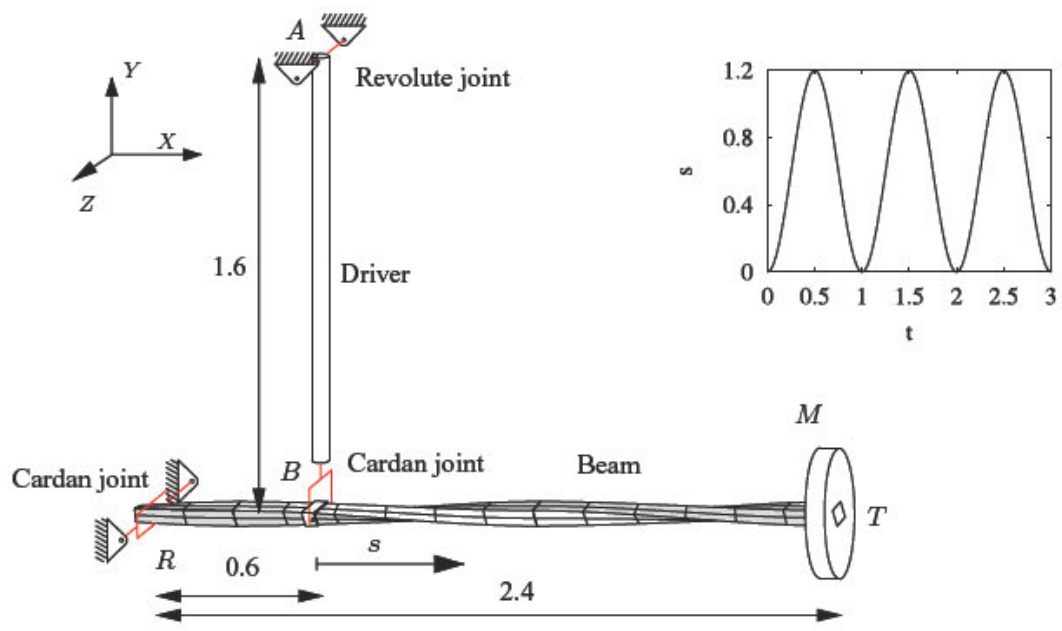

Fig. 10 Geometry and applied released displacement of the driven screw joint problem

twisted at the same ratio of the screw joint in such a way that at point $R$ the local axes $Y$ and $Z$ of the beam are rotated by $30^{\circ}$ with respect to the global axis $Y$ and $Z$ while at point $T$ they are rotated by $-30^{\circ}$ with respect to the same global axes. At point $T$ a rigid body $M$ is attached to the beam as depicted in Figure 10. The beam is attached to a fixed point $R$ by means of a universal joint which has the rotation around the $X$ axis constrained. The geometric and material properties of the beams are given in Figure 10 and Table 2. We note that although this problem may not necessarily represent a real physical device, it exploits the use of joints we are dealing with and, most importantly, allows us to compare the method of Lagrange multipliers [4], where this problem was originally given, with our approach. Although the two procedures are very different, they lead to remarkably similar outputs.

The displacement of the screw joint is prescribed during the analysis according to the function $r_{R}=0.6(1-\cos 2 \pi t) \mathbf{g}_{r}$, where $t$ is the time variable and $\mathbf{g}_{r}$ is the body-attached axis perpendicular to the cross-section of the beam at point $B$ for the NN approach, or the tangent to the centroid line in the NE approach (in both cases, initially in the direction of the global axis $X$ ).

The driver and the beam have been discretised using 2 and 12 quadratic elements respectively and the total response time is 3 seconds. The use of twelve elements along the screw beam is not necessary for convergence reasons, but it is rather required to model the geometrical properties of the beam more accurately.

We have run the problem with the NN approach described in [22] using an energymomentum or $\beta$ algorithm. We have compared these results with the NE approach described in [21] (trapezoidal rule) and the SMI and SI algorithms presented here. We remark that although Bauchau and Bottasso [4] employed an energy decaying scheme with a variable time-step, we employed a time-step $\Delta t=0.01$ that is ten times larger than their maximum value [6].

Pairs of Figures 11, 12 and 13, 14 show the out-of-plane displacement $u_{z}$ and the rotation $\theta_{X}$ of the tip point $T$ given in [4], and for the time integration schemes $\beta$ and SI, respectively. They agree very nicely apart from some small differences during the last second of the simulation, which we believe is due to the dissipative character of the time-integration scheme used in [4] and the different spatial and time discretisation used. 
Table 2 Geometrical and material properties of the driven screw joint problem (all values expressed with the units $\mathrm{kN}, \mathrm{m}$ and $\mathrm{kg}$ )

\begin{tabular}{|c|c|c|c|c|c|c|c|c|}
\hline & $E A$ & $E I_{y y}$ & $E I_{z z}$ & $G J$ & $G A_{y}$ & $G A_{z}$ & $\rho I_{y y}$ & $\rho I_{z z}$ \\
\hline Driver & 44000 & 23 & 300 & 28 & 14000 & 2800 & 0.001 & 0.011 \\
\hline \multirow[t]{3}{*}{ Beam } & 44000 & 300 & 23 & 28 & 2800 & 14800 & 0.001 & 0.011 \\
\hline & & & $\mathrm{M}[\mathrm{kg}]$ & $\rho I_{y y}$ & $\rho I_{z z}$ & & & \\
\hline & & Tip mass $\mathrm{M}$ & 40 & 0.225 & 0.225 & & & \\
\hline
\end{tabular}

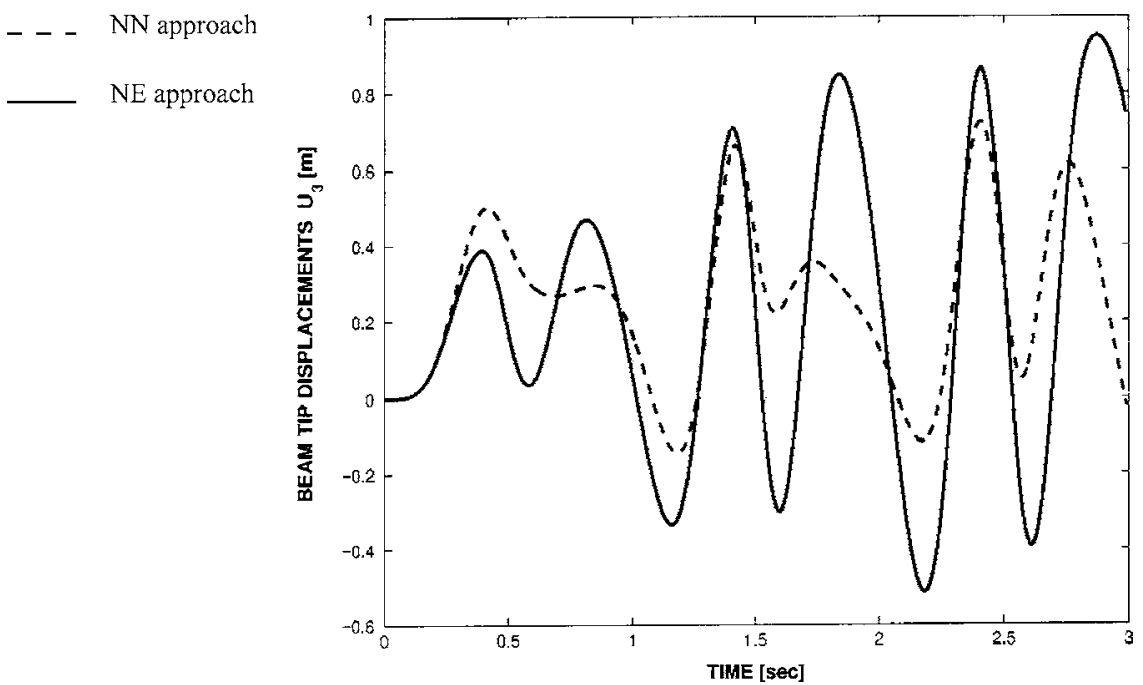

Fig. 11 Out-of-plane displacement $u_{z}$ of the tip of the beam for the driven screw joint problem in [4]

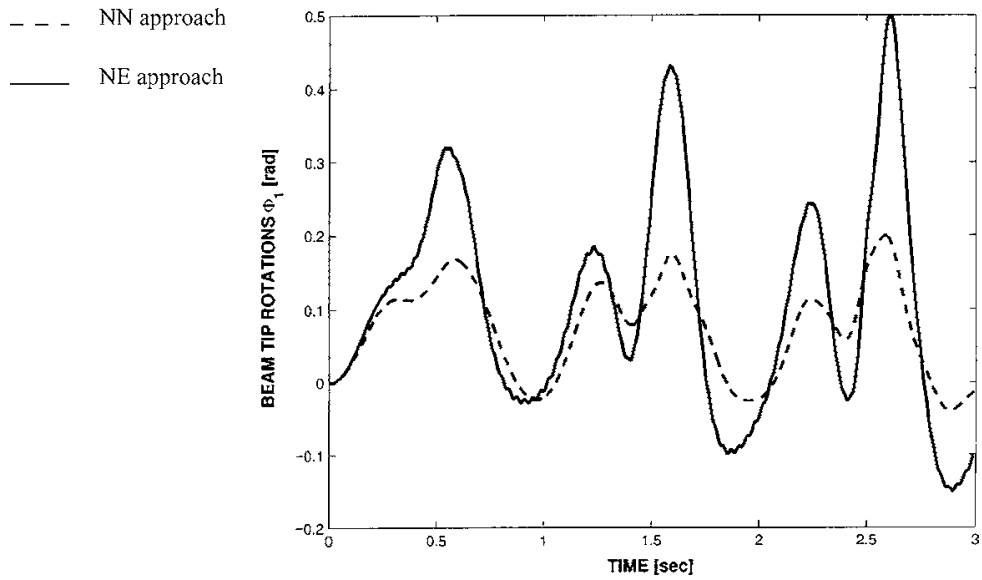

Fig. 12 Rotation $\theta_{x}$ of the tip of the beam for the driven screw joint problem in [4] 


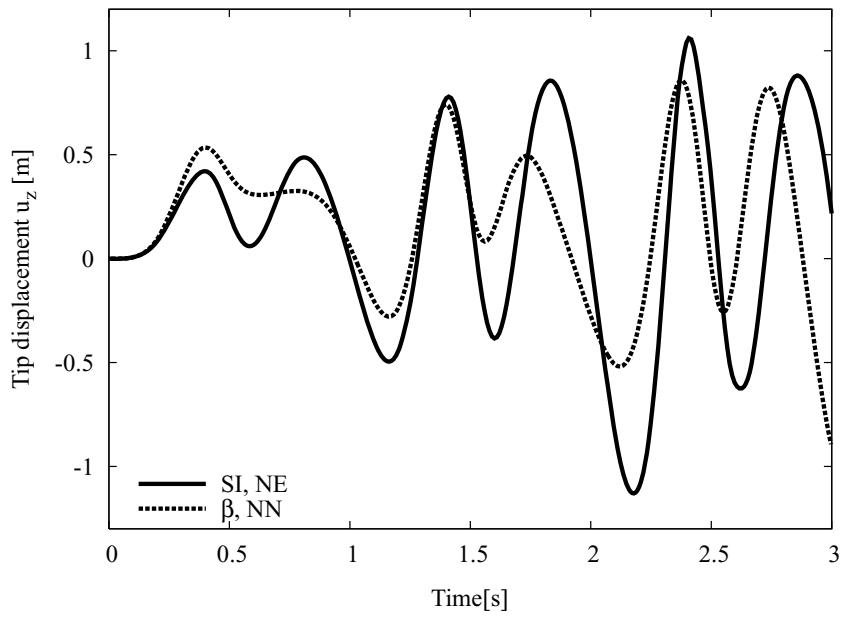

Fig. 13 Out-of-plane displacement $u_{z}$ of the tip of the beam for the driven screw joint problem using the $\beta$ (NN approach) and SMI (NE approach) algorithms $(\Delta t=0.005)$

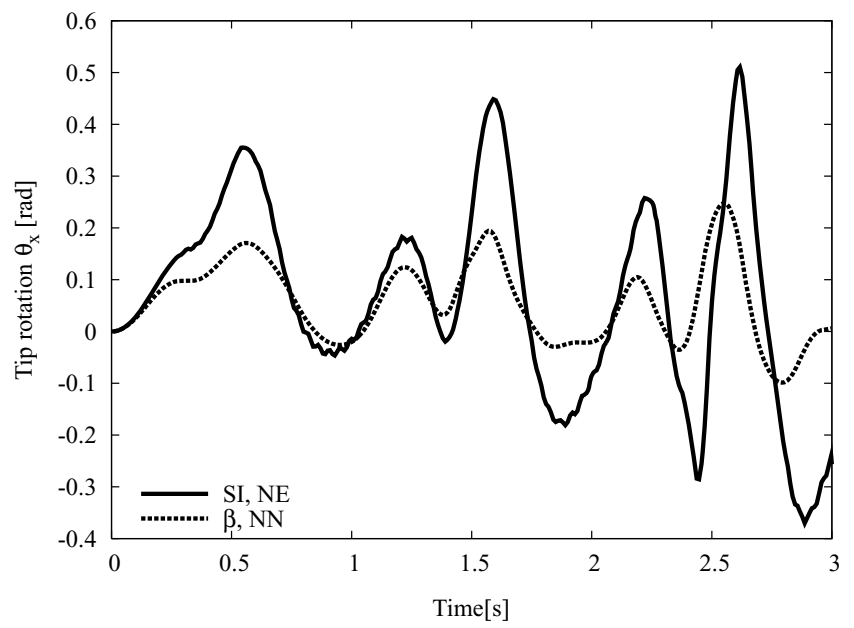

Fig. 14 Rotation $\theta_{x}$ of the tip of the beam for the driven screw joint problem using algorithm $\beta$ (NN approach) and algorithm SI (NE approach) $(\Delta t=0.005)$

A constant time-step $\Delta t=0.005$ was initially used, and all the algorithms completed the analysis successfully. In a second set of runs, with the results shown in Figures 15 and 16 , a time-step $\Delta t=0.01$ was employed. With this time-step size, and when using the NN approach, the trapezoidal rule required one time-step halving, whereas the $\beta$ algorithm successfully terminated the analysis with a constant time-step. With regard to the NE approach, the trapezoidal rule and algorithm SI required three and one time-step halvings, respectively, whereas SMI failed to converge after a series of successive time-step reductions. This is in agreement with the conclusions drawn from the previous example, where it was shown that algorithm SMI is visibly less robust than algorithm SI. It is also worth pointing out that the $\mathrm{NN}$ and NE formulations are meant to model two physically different problems with very different characteristics. The present discrepancy between the results of these two approaches 


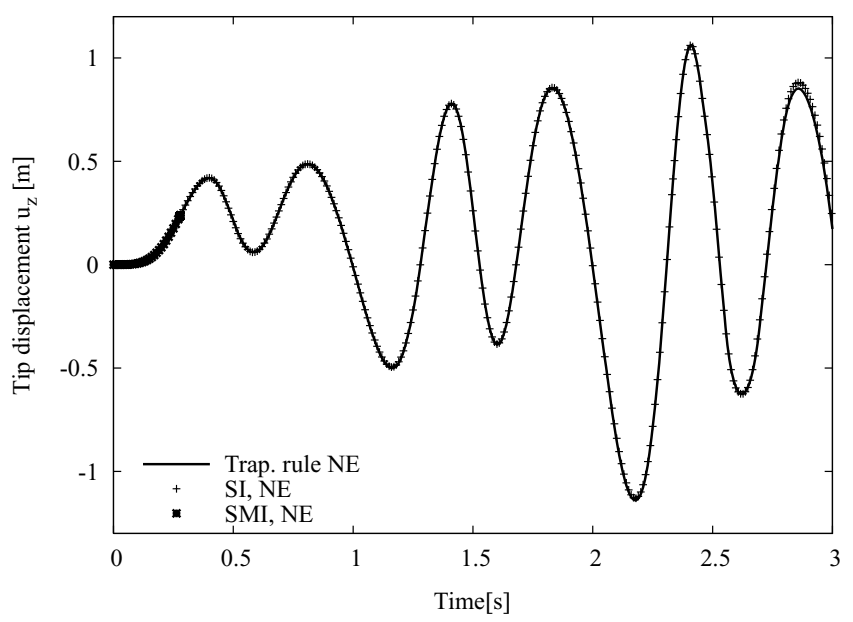

Fig. 15 Out-of-plane displacement $u_{z}$ of the tip of the beam for the driven screw joint problem using the NE $\operatorname{approach}(\Delta t=0.01)$

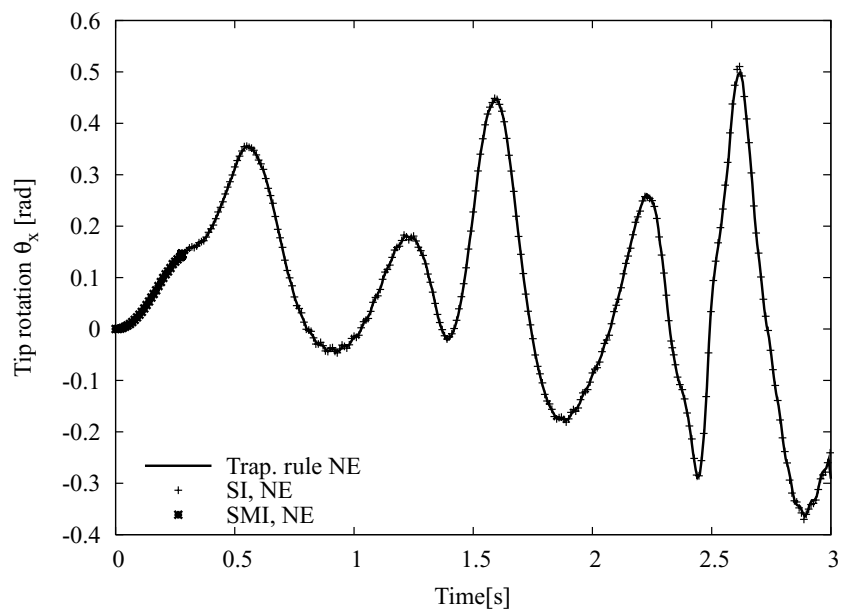

Fig. 16 Rotation $\theta_{x}$ of the tip of the beam for the NE approach $(\Delta t=0.01)$

underlines the unsuitability of using the NN approach in a problem with a deformable slideline. From the computational point of view, modelling the sliding joint (NE approach) is considerably more demanding than modelling the prismatic joint of the $\mathrm{NN}$ approach.

Figure 17 shows the three components of the total angular momentum of the structure. Although the trapezoidal rule and the SI algorithm show different convergence properties, this differences are not reflected in the values of the total angular momentum, which are very minimal.

\section{Discussion and conclusions}

The paper has analysed the sliding contact in 3D beams using the master-slave approach by means of an incrementally-based family of algorithms. It has been demonstrated that 


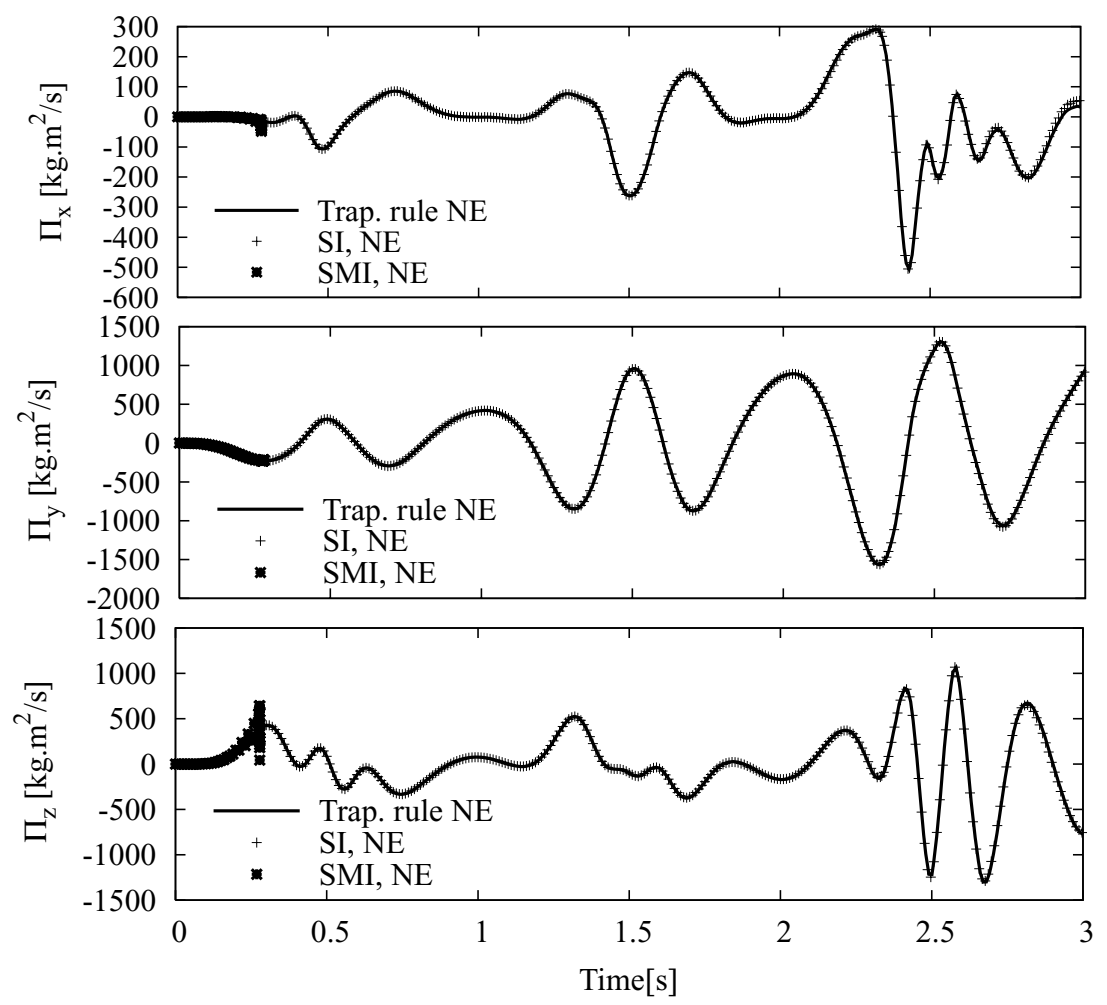

Fig. 17 Three components of the angular momentum for the NE approach and $\Delta t=0.01$

the use of constraint equations can be avoided by embedding the contact conditions in the equilibrium equations.

Special attention has been dedicated to the exact satisfaction of the sliding conditions, and the conservation of constants of motion, in particular the angular momentum. For the algorithms used here, there exists a conflict between the two. This has led to two algorithms, of which the first one (SMI) is angular momentum-conserving, but it satisfies the constraint kinematics only approximately, while the second one (SI) satisfies the constraint kinematics exactly, but only at the expense of the angular momentum conservation. The numerical tests show that the latter provides more robust results. This may appear in contradiction with some of our earlier results, where the exact conservation of angular momentum was emphasised as a key point. Obviously, such a conclusion still holds true for the problems where the momentum conservation does not incur constraint violation. For the problems where the exact satisfaction of both requirements within a given setup is not viable, however, the results of this work indicate that the satisfaction of constraint conditions is to be taken as a more important requirement than the angular momentum conservation. The approximation of constraint kinematics in SMI, namely, introduces an additional amount of energy error in this algorithm, which makes it more susceptible to a sudden energy blow-up.

It has been also shown that both algorithms can be easily modified to model joints with dependent released degrees of freedoms such as the screw-joints, cam joints and rack-andpinion joints. Other time-integration strategies are possible, constructed from the same or similar underlying time-integration algorithms for beams. 


\section{Appendix A: Proof of the conservation of momenta}

We will find the necessary conditions for the conservation of momenta using algorithm SMI without contact transition, i.e. considering the two-element model shown in Figure 1 in conjunction with the resulting equilibrium Equations in (30). Since we aim to prove the conservation of angular momentum in the absence of applied loads, we will assume $\boldsymbol{g}_{e}^{I, i}=\mathbf{0}$, $i=1, \ldots, N_{I}, I=A, B$. We will start with a general expression for matrix $\mathbf{A}$, and then prove that the condition $\mathbf{A}=\mathbf{I}$ in the master-slave relation for rotations is necessary for the conservation of angular momentum. This implies the use of the following form of the interpolation matrix $\overline{\mathbf{I}}_{X \gamma}^{j}$ in (28b):

$$
\overline{\mathbf{I}}_{X}^{j}=\left[\begin{array}{cc}
I_{X \gamma}^{j} \mathbf{I} & \mathbf{0} \\
\mathbf{0} & I_{X_{n+1}}^{j} \mathbf{A}
\end{array}\right] .
$$

Keeping this in mind and splitting the residual vector into its force and moment parts, i.e. $\boldsymbol{g}^{I, i}=\left\{\boldsymbol{g}_{f}^{I, i} \boldsymbol{g}_{\phi}^{I, i}\right\}$, the equilibrium equations (30) are written as

$$
\begin{array}{rl}
\mathbf{R}^{\mathrm{T}} \boldsymbol{g}^{A, N_{A}}=\mathbf{0} & \\
\boldsymbol{g}^{A, i}=\mathbf{0} & i=1, \ldots, N_{A}-1 \\
\boldsymbol{g}_{f}^{B, j}+I_{X \gamma}^{j} \boldsymbol{g}_{f}^{A, N_{A}}=\mathbf{0} & j=1, \ldots, N_{B} \\
\boldsymbol{g}_{\phi}^{B, j}+I_{X_{n+1}}^{j} \mathbf{A g}_{\phi}^{A, N_{A}}=\mathbf{0} & j=1, \ldots, N_{B} .
\end{array}
$$

On the other hand, by adding all the nodal load vectors for both elements, using Equations (38b) and (38c) we get

$$
\begin{aligned}
\sum_{i=1}^{N_{A}} \boldsymbol{g}_{f}^{A, i}+\sum_{j=1}^{N_{B}} \boldsymbol{g}_{f}^{B, j} & =\boldsymbol{g}_{f}^{A, N_{A}}-\underbrace{\left(\sum_{j=1}^{N_{B}} I_{X \gamma}^{j}\right)}_{=1} \boldsymbol{g}_{f}^{A, N_{A}}=\mathbf{0}, \\
\sum_{i=1}^{N_{A}} \boldsymbol{g}_{\phi}^{A, i}+\sum_{j=1}^{N_{B}} \boldsymbol{g}_{\phi}^{B, j} & =\boldsymbol{g}_{\phi}^{A, N_{A}}-\left(\sum_{j=1}^{N_{B}} I_{X_{n+1}}^{j}\right) \mathbf{A} \boldsymbol{g}_{\phi}^{A, N_{A}} \\
& =(\mathbf{I}-\mathbf{A}) \boldsymbol{g}_{\phi}^{A, N_{A}},
\end{aligned}
$$

where the completeness condition of the Lagrangian polynomials $I_{X \gamma}^{j}$ and $I_{X_{n+1}}^{j}$ has been utilised. The conservation of the total linear momentum $\boldsymbol{L}=\int_{L} \rho A \dot{\boldsymbol{r}} d X$ can be proved by noting that from the definitions of the contributions to the residual vector in (7) we have

$$
\begin{aligned}
\Delta \boldsymbol{L} & =\int_{L^{A}+L^{B}} \rho A\left(\dot{\boldsymbol{u}}_{n+1}-\dot{\boldsymbol{u}}_{n}\right) d X \\
& =\int_{L^{A}+L^{B}}(\rho A\left(\dot{\boldsymbol{u}}_{n+1}-\dot{\boldsymbol{u}}_{n}\right)+\underbrace{\left(\sum_{i=1}^{N_{A}+N_{B}} I^{i^{\prime}}\right)}_{=0} \boldsymbol{\Lambda}_{n+\frac{1}{2}} \boldsymbol{N}_{n+\frac{1}{2}}) d X \\
& =\sum_{i=1}^{N_{A}} \boldsymbol{g}_{f}^{A, i}+\sum_{j=1}^{N_{B}} \boldsymbol{g}_{f}^{B, j}=\mathbf{0},
\end{aligned}
$$


where the last identity follows from Equation (39a). In order to ease the forthcoming derivations, let us set $\boldsymbol{\pi}=\rho \mathbf{\Lambda} \mathbf{J W}$ as the specific local angular momentum. The conservation of the total angular momentum $\Pi=\int_{L}(\boldsymbol{\pi}+\rho A \widehat{\boldsymbol{r}} \dot{\boldsymbol{r}}) d X$ can be proved by first remarking that from the definitions of the contributions to the residual vector (7), completeness of the Lagrangian polynomials and Equation (39b) we have

$$
\frac{1}{\Delta t} \int_{L^{A}+L^{B}} \Delta \pi d X-\int_{L^{A}+L^{B}} \widehat{\boldsymbol{r}}_{n+\frac{1}{2}}^{\prime} \boldsymbol{\Lambda}_{n+\frac{1}{2}} \mathbf{N}_{n+\frac{1}{2}} d X=(\mathbf{I}-\mathbf{A}) \mathbf{g}^{A, N_{A}} .
$$

Hence, the increment of the angular momentum $\Delta \Pi$ over a time-step is given by

$$
\begin{aligned}
\Delta \boldsymbol{\Pi} & =\int_{L^{A}+L^{B}}\left(\Delta \boldsymbol{\pi}+\rho A \widehat{\boldsymbol{r}}_{n+1} \dot{\boldsymbol{r}}_{n+1}-\rho A \widehat{\boldsymbol{r}}_{n} \dot{\boldsymbol{r}}_{n}\right) d X \\
& =\int_{L^{A}+L^{B}} \Delta \boldsymbol{\pi} d X+\int_{L^{A}+L^{B}} \rho A\left(\widehat{\boldsymbol{r}}_{n+\frac{1}{2}}\left(\dot{\boldsymbol{u}}_{n+1}-\dot{\boldsymbol{u}}_{n}\right)+\widehat{\boldsymbol{u}} \dot{\boldsymbol{r}}_{n+\frac{1}{2}}\right) d X \\
& =\int_{L^{A}+L^{B}} \Delta \boldsymbol{\pi} d X+\int_{L^{A}+L^{B}} \rho A \widehat{\boldsymbol{r}}_{n+\frac{1}{2}} \dot{\boldsymbol{u}} d X,
\end{aligned}
$$

where the last result follows from the time-integration mid-point rule (5), i.e. $\widehat{u} \dot{\boldsymbol{r}}=\mathbf{0}$. In addition, inserting Equation (40) into the first integral of (41) yields

$$
\begin{aligned}
\Delta \boldsymbol{\Pi}= & \Delta t \int_{L^{A}+L^{B}} \widehat{\boldsymbol{r}}_{n+\frac{1}{2}}^{\prime} \boldsymbol{\Lambda}_{n+\frac{1}{2}} \mathbf{N}_{n+\frac{1}{2}} d X \\
& +\int_{L^{A}+L^{B}} \rho A \widehat{\boldsymbol{r}}_{n+\frac{1}{2}}\left(\dot{\boldsymbol{u}}_{n+1}-\dot{\boldsymbol{u}}_{n}\right) d X+\Delta t(\mathbf{I}-\mathbf{A}) \boldsymbol{g}_{\phi}^{A, N_{A}} \\
= & \Delta t \sum_{i}^{N_{A}} \widehat{\boldsymbol{r}}_{i, n+\frac{1}{2}} \int_{L^{A}} I^{i \prime} \boldsymbol{\Lambda}_{n+\frac{1}{2}} \mathbf{N}_{n+\frac{1}{2}} d X \\
& +\Delta t \sum_{j}^{N_{B}} \widehat{\boldsymbol{r}}_{j, n+\frac{1}{2}} \int_{L^{B}} I^{j^{\prime \prime}} \boldsymbol{\Lambda}_{n+\frac{1}{2}} \mathbf{N}_{n+\frac{1}{2}} d X \\
& +\int_{L^{A}+L^{B}} \rho A \widehat{\boldsymbol{r}}_{n+\frac{1}{2}}\left(\dot{\boldsymbol{u}}_{n+1}-\dot{\boldsymbol{u}}_{n}\right) d X+\Delta t(\mathbf{I}-\mathbf{A}) \boldsymbol{g}_{\phi}^{A, N_{A}},
\end{aligned}
$$

where the interpolation $\boldsymbol{r}_{n+\frac{1}{2}}^{\prime}=I^{i \prime} \boldsymbol{r}_{n+\frac{1}{2}}^{i}$ within each element has been used in the last step. By recalling the definitions of the force part of $\boldsymbol{g}^{i}=\boldsymbol{g}_{d}^{i}+\boldsymbol{g}_{v}^{i}$ in (7), the first two terms in the last result of (42) are expressible as

$$
\begin{aligned}
& \Delta t \sum_{i}^{N_{A}} \widehat{\boldsymbol{r}}_{i, n+\frac{1}{2}}\left(\boldsymbol{g}_{f}^{A, i}-\frac{1}{\Delta t} \int_{L^{A}} \rho A I^{i}\left(\dot{\boldsymbol{u}}_{n+1}-\dot{\boldsymbol{u}}_{n}\right) d X\right) \\
& \quad+\Delta t \sum_{j}^{N_{B}} \widehat{\boldsymbol{r}}_{j, n+\frac{1}{2}}\left(\boldsymbol{g}_{f}^{B, j}-\frac{1}{\Delta t} \int_{L^{B}} \rho A I^{j}\left(\dot{\boldsymbol{u}}_{n+1}-\dot{\boldsymbol{u}}_{n}\right) d X\right) \\
& =\Delta t \widehat{\boldsymbol{r}}_{A, N_{A}, n+\frac{1}{2}} \boldsymbol{g}_{f}^{A, N_{A}}-\Delta t \sum_{j}^{N_{B}} \widehat{\boldsymbol{r}}_{j, n+\frac{1}{2}} I_{X \gamma}^{j} \boldsymbol{g}_{f}^{A, N_{A}} \\
& \quad-\int_{L^{A}+L^{B}} \rho A \widehat{\boldsymbol{r}}_{n+\frac{1}{2}}\left(\dot{\boldsymbol{u}}_{n+1}-\dot{\boldsymbol{u}}_{n}\right) d X,
\end{aligned}
$$


where the force part of the equilibrium equations (38b) and (38c) have been used in the last identity. Hence, inserting this result into (42), and using the definitions in (16) yields

$$
\begin{aligned}
\Delta \boldsymbol{\Pi}= & \Delta t\left(\widehat{\boldsymbol{r}}_{A, N_{A}, n+\frac{1}{2}}-\sum_{j}^{N_{B}} I_{X \gamma}^{j} \widehat{\boldsymbol{r}}_{j, n+\frac{1}{2}}\right) \boldsymbol{g}_{f}^{A, N_{A}}+\Delta t(\mathbf{I}-\mathbf{A}) \boldsymbol{g}_{\phi}^{A, N_{A}} \\
= & \frac{\Delta t}{2}\left(\sum_{k}^{N_{B}} I_{X_{n+1}}^{k} \widehat{\boldsymbol{r}}_{k, n+1}+\sum_{l}^{N_{B}} I_{X_{n}}^{l} \widehat{\boldsymbol{r}}_{l, n}-\sum_{j}^{N_{B}} I_{X \gamma}^{j}\left(\widehat{\boldsymbol{r}}_{j, n}+\widehat{\boldsymbol{r}}_{j, n+1}\right)\right) \boldsymbol{g}_{f}^{A, N_{A}} \\
& +\Delta t(\mathbf{I}-\mathbf{A}) \boldsymbol{g}_{\phi}^{A, N_{A}} \\
= & \frac{\Delta t}{2}\left(\gamma \widehat{\boldsymbol{u}}_{t_{n+1}}-(1-\gamma) \widehat{\boldsymbol{u}}_{t_{n}}\right) \boldsymbol{g}_{f}^{A, N_{A}}+\Delta t(\mathbf{I}-\mathbf{A}) \boldsymbol{g}_{\phi}^{A, N_{A}} .
\end{aligned}
$$

It is clear that $\mathbf{A}=\mathbf{I}$, together with the kinematic condition $\gamma \boldsymbol{u}_{t_{n+1}}-(1-\gamma) \boldsymbol{u}_{t_{n}}=\mathbf{0}$, render the algorithm angular-momentum conserving.

Acknowledgements The numerical results in this work have been obtained using the research version of the finite element code LUSAS, kindly provided by FEA Ltd. Financially supported by the Engineering and Physical Sciences Research Council under grants GR/R 04171/01 and AF/100089.

\section{References}

1. Argyris, J.H.: An excursion into large rotations. Comp. Meth. Appl. Mech. Engng. 32, 81-155 (1982)

2. Armero, F., Petőcz, E.: Formulation and analysis of conserving algorithms for frictionless dynamic contact/impact problems. Comp. Meth. Appl. Mech. Engng. 158, 269-300 (1998)

3. Bauchau, O.: On the modeling of prismatic joints in flexible multi-body systems. Comp. Meth. Appl. Mech. Engng. 181, 87-105 (2000)

4. Bauchau, O., Bottasso, C.L.: Contact conditions for cylindrical, prismatic, and screw joints in flexible multibody systems. Multibody System Dyn. 5, 251-278 (2001)

5. Betsch, P.: The discrete null space method for the energy consistent integration of constrained mechanical systems. Part I: Holonomic constraints. Comp. Meth. Appl. Mech. Engng. 194, 5159-5190 (2005)

6. Bottasso, C.L.: Personal communication (2002).

7. Cardona, A., Géradin, M.A.: A beam finite element non-linear theory with finite rotations. Int. J. Num. Meth. Engng. 26, 2403-2438 (1988)

8. Crisfield, M.A., Jelenić, G.: Objectivity of strain measures in the geometrically exact three-dimensional beam theory and its finite-element implementation. Proc. Royal Soc. London 455, 1125-1147 (1999)

9. Géradin, M.A., Cardona, A.: Flexible multibody dynamics. A Finite Element Approach. John Wiley \& Sons (2001)

10. Ibrahimbegović, A., Mamouri, S.: On rigid components and joint constraints in nonlinear dynamics of flexible multibody systems employing 3D geometrically exact beam model. Comp. Meth. Appl. Mech. Engng. 188, 805-831 (2000)

11. Jelenić, G., Crisfield, M.A.: Non-linear master-slave relationships for joints in 3D beams with large rotations. Comp. Meth. Appl. Mech. Engng. 135, 211-228 (1996)

12. Jelenić, G., Crisfield, M.A.: Geometrically exact 3D beam theory: implementation of a strain-invariant finite element for statics and dynamics. Comp. Meth. Appl. Mech. Engng. 171, 141-171 (1999)

13. Jelenić, G., Crisfield, M.A.: Stability and convergence characteristics of conserving algorithms for dynamics of 3D rods. Technical report, Department of Aeronautics, Imperial College, London (1999)

14. Jelenić, G., Crisfield, M.A.: Dynamic analysis of 3D beams with joints in presence of large rotations. Comp. Meth. Appl. Mech. Engng. 190, 4195-4230 (2001)

15. Jelenić, G., Crisfield, M.A.: Problems associated with the use of Cayley transform and tangent scaling for conserving energy and momenta in the Reissner-Simo beam theory. Comm. Num. Meth. Engng. 18, $711-720(2002)$ 
16. Laursen, T.A., Chawla, V.: Design of energy conserving algorithms for frictionless dynamic contact problems. Int. J. Num. Meth. Engng. 40, 863-886 (1997)

17. Leyendecker, S., Betsch, P., Steinmann, P.: Objective energy-momentum conserving integration for the constrained dynamics of geometrically exact beams. Comp. Meth. Appl. Mech. Engng. 195, 2131-2333 (2006)

18. Marjamäki, H., Mäkinen, J.: Modelling telescopic boom - The plane case: Part I. Comput. Struct. 81, $1597-1609$ (2003)

19. Mitsugi, J.: Direct strain measure for large displacement analyses on hinge connected beam structures. Comput. Struct. 64, 509-517 (1997)

20. Muñoz, J.: Finite-element analysis of flexible mechanisms using the master-slave approach with emphasis on the modelling of joints. PhD thesis, Imperial College London (2004)

21. Muñoz, J., Jelenić, G.: Sliding contact conditions using the master-slave approach with application on the geometrically non-linear beams. Int. J. Solids Struct. 41, 6963-6992 (2004)

22. Muñoz, J., Jelenić, G., Crisfield, M.: Master-slave approach for the modelling of joints with dependent degrees of freedom in flexible mechanisms. Comm. Num. Meth. Engng. 19, 689-702 (2003)

23. Romero, I., Armero, F.: An objective finite element approximation of the kinematics of geometrically exact rods and its use in the formulation of an energy-momentum conserving scheme in dynamics. Int. J. Num. Meth. Engng. 54, 1683-1716 (2002)

24. Simo, J.C.: A finite strain beam formulation. The three dimensional dynamic problem. Part I. Comp. Meth. Appl. Mech. Engng. 49, 55-70 (1985)

25. Simo, J.C., Tarnow, N., Doblare, M.: Non-linear dynamics of three-dimensional rods: exact energy and momentum conserving algorithms. Int. J. Num. Meth. Engng. 38, 1431-1473 (1995)

26. Wriggers, P.: Computational Contact Mechanics, Wiley (2002) 